\title{
Brouwer's Weak Counterexamples and the Creative Subject: A Critical Survey
}

\section{Peter Fletcher $^{1}$ iD}

Received: 21 January 2019 / Accepted: 11 February 2020 / Published online: 9 May 2020

(C) The Author(s) 2020

\begin{abstract}
I survey Brouwer's weak counterexamples to classical theorems, with a view to discovering (i) what useful mathematical work is done by weak counterexamples; (ii) whether they are rigorous mathematical proofs or just plausibility arguments; (iii) the role of Brouwer's notion of the creative subject in them, and whether the creative subject is really necessary for them; (iv) what axioms for the creative subject are needed; (v) what relation there is between these arguments and Brouwer's theory of choice sequences. I refute one of Brouwer's claims with a weak counterexample of my own. I also examine Brouwer's 1927 proof of the negative continuity theorem, which appears to be a weak counterexample reliant on both the creative subject and the concept of choice sequence; I argue that it provides a good justification for the weak continuity principle, but it is not a weak counterexample and it does not depend essentially on the creative subject.
\end{abstract}

Keywords Brouwer - Intuitionistic analysis - Intuitionistic logic ·

Weak counterexamples $\cdot$ Creative subject $\cdot$ Choice sequences

\section{Introduction}

Between 1923 and 1955 Brouwer published a series of arguments, known as weak counterexamples, intended to show that certain classical theorems are not provable intuitionistically. (He also gave strong counterexamples, from 1928 onwards [9], but these are outside the scope of my study.) Here is a typical example. We choose a fleeing property $P$, that is, a decidable property $P$ of natural numbers for which we lack a proof of $\exists n P(n)$ or $\neg \exists n P(n)$. (His first examples [5, 6] used a property $P$ based on occurrence of the digit sequence 0123456789 in the decimal expansion of

Peter Fletcher

p.fletcher@keele.ac.uk

1 School of Computing and Mathematics, Keele University, Keele, ST5 2BG, UK 
$\pi$; he later generalised to an arbitrary fleeing property.) Using $P$ we construct a real number $x$ and show that

$$
x=0 \vee x<0 \vee x>0 \Rightarrow \exists n P(n) \vee \neg \exists n P(n) .
$$

If we could prove $x=0 \vee x<0 \vee x>0$ then we could thereby prove $\exists n P(n) \vee$ $\neg \exists n P(n)$ - which by hypothesis we cannot. Hence we cannot prove $x=0 \vee$ $x<0 \vee x>0$. This is a weak counterexample to the classical theorem $\forall x \in$ $\mathbb{R}(x=0 \vee x<0 \vee x>0)$. It is called 'weak' because it depends on the accidental and possibly temporary fact that we lack a proof of $\exists n P(n)$ or $\neg \exists n P(n)$. We may discover such a proof tomorrow; but in that case we can always choose another fleeing property $P^{\prime}$ and construct a new $x^{\prime}$ for which we cannot prove $x^{\prime}=$ $0 \vee x^{\prime}<0 \vee x^{\prime}>0$. We shall never run out of fleeing properties, since we shall never solve all $\Sigma_{1}$ problems, so we shall always have a counterexample.

What is the status of such arguments? Are they genuine mathematical proofs or merely plausibility arguments (as van Atten [39] calls them)? Has anything really been proved? It is disconcerting to see a 'proof' that is dependent on a contingent and continually changeable choice of fleeing property. A property may be fleeing from one person's point of view but not from another person's, if the latter possesses a proof of $\exists n P(n)$ or $\neg \exists n P(n)$.

I shall argue that these arguments are nevertheless rigorous proofs of precise theorems, which are a permanent and objective addition to our mathematical knowledge. To this end I shall reformulate them slightly to make them apply to all decidable properties of natural numbers, not just fleeing ones (see $\S 4$ ).

A second kind of weak counterexample, first introduced in Brouwer's Berlin lectures of 1927 [42], uses the theory of the creative (or creating) subject. Choose a proposition $A$ that is not judgeable (i.e., we cannot at present prove $A$ or $\neg A$ ). Using $A$ we construct a real number $x$ and show that, e.g.,

$$
x=0 \vee x<0 \vee x>0 \Rightarrow A \vee \neg A .
$$

If we could prove $x=0 \vee x<0 \vee x>0$ then we could thereby prove $A \vee \neg A$ - which by hypothesis we cannot. Hence we cannot prove $x=0 \vee x<0 \vee x>$ 0 .

Sometimes Brouwer begins instead with a proposition that is not testable (i.e., we cannot at present prove $\neg A \vee \neg \neg A$ ) and constructs $x$ such that, e.g.,

$$
x=0 \vee x<0 \vee x>0 \Rightarrow \neg A \vee \neg \neg A .
$$

Such weak counterexamples only work as long as $A$ remains unjudgeable (or untestable). As soon as we obtain a proof of $A \vee \neg A$ (or $\neg A \vee \neg \neg A$ ), $x$ ceases to be a counterexample and we must choose another $A$, giving another $x$. We shall never run out of unjudgeable (or untestable) propositions, so we shall always have a counterexample.

Again, I claim that these arguments are genuine mathematical theorems, though I shall reformulate them to apply to all propositions, rather than to unjudgeable or untestable ones (see $\S 4$ ); here, however, the arguments are dependent on accepting the theory of the creative subject. This involves reasoning about one's own future proving activity; the creative subject is oneself, viewed under certain idealisations. In 
Brouwer's arguments the creative subject is imagined to be choosing the terms of an infinite sequence while simultaneously proving various theorems; any theorem that is proved between the choice of the $(n-1)$ th and $n$th terms is said (by Brouwer's commentators) to be proved at stage $n$. We write $\vdash_{n} A$ to mean that the creative subject will possess a proof of a proposition $A$ at stage $n$. It is usually asserted that $A$ holds iff there exists $n$ such that $\vdash_{n} A$ (or that $A$ is false iff there is no such $n$; see $\S 3$ ).

The theory of the creative subject is considered philosophically dubious by most intuitionists because

(i) it makes mathematics dependent upon time and the person who is the creative subject;

(ii) it makes mathematics dependent on contingent factors;

(iii) there is an obvious risk of self-referential paradoxes in defining constructions that depend on one's own future constructive activity (e.g., Troelstra's paradox [36]);

(iv) if one assumes that only finitely many propositions are proved at each stage then all the provable propositions can be enumerated, yet the species of provable propositions is surely an example of what Brouwer [3] called a denumerably unfinished set.

I shall not discuss the merits of the creative subject but shall simply consider how it is used. I shall examine Brouwer's weak counterexamples, and one due to Heyting [27], stating each one with and without the creative subject, in order to isolate the contribution that the creative subject makes.

A very odd feature of the theory of the creative subject is the fact that it has developed separately from Brouwer's theory of choice sequences, which is the basis for intuitionistic analysis. Both theories draw on the same idea: an infinite sequence of possibly constrained choices, viewed as a single intensional process. There was no distinction between them in Brouwer's mind. Nevertheless, they diverged in the work of later intuitionists. As Niekus remarks,

No connection has been made between the standard theories of choice sequences and the TCS [the theory of the creative subject]; the two theories have been developed completely separately. In the intuitionistic handbooks, a considerable amount of space is reserved for the theories on choice sequences, while the method of the creating subject is treated in the last few pages. See for example Dummett 1977 [26] and van Dalen and Troelstra 1988 [32, 38].

For Niekus the so-called theory of the creative subject is simply the application of particular choice sequences, as opposed to the theory of choice sequences in general. There are two reasons why the two theories have developed separately. One is that the theory of the creative subject 'appears constantly to tremble on the edge of absurdity or paradox' [26, p359], and it has been felt to be prudent to insulate choice sequences and intuitionistic analysis from its obscurities; the other is that the two theories need different axioms and naturally pull in different directions. There is one place in Brouwer's writings where the two theories seem to overlap: this is his 1927 proof that all functions are negatively continuous. There is also a 'conditional checking-number' argument in 1954, which van Atten [39, appendix B] sees 
as resembling the negative continuity proof. Both arguments are therefore of special interest and I shall examine them in $\$ 9$. I shall show that the 1927 negative continuity argument can be understood in terms of the creative subject, but it can also be understood in an atemporal way in terms of Appleby's [1] concept of 'knowledge states', which provides an alternative view of the foundations of intuitionistic analysis. The 'conditional checking-number' claim of 1954 I shall refute, using a weak counterexample argument of my own.

The general tendency of this paper is to show that time, subjectivity and contingency play a much less essential role in intuitionistic mathematics than they appear to. No doubt they played a central role in Brouwer's thought, but the mathematics can be restated without them.

\section{Preliminaries}

- I use ' $x<y$ ' to denote the positive order relation on real numbers, in accordance with modern usage (see definition below); Brouwer usually calls it ' $x<0 \quad y$ '.

- ' $x \leq y$ ' means $\neg y<x$, as usual.

- I use ' $x \not y$ ' for the negative order relation, defined by

$$
x \not y \Leftrightarrow \neg \neg x<y \Leftrightarrow x \leq y \wedge x \neq y
$$

which Brouwer usually writes simply as ' $x<y$ '.

- $\quad x$ \# $y$ means that $x$ is apart from $y$, i.e., $x<y \vee y<x$.

- $\mathbb{Q}$ is the set of rational numbers; $x \notin \mathbb{Q}$ means $\neg x \in \mathbb{Q} ; x \notin \mathbb{Q}$ means $\neg \neg x \in \mathbb{Q}$.

When I need to use a specific representation of the real numbers I shall consider a real number as defined by a nested infinite sequence of binary intervals

$$
\left[\frac{x_{0}-1}{2^{0}}, \frac{x_{0}+1}{2^{0}}\right] \supset\left[\frac{x_{1}-1}{2^{1}}, \frac{x_{1}+1}{2^{1}}\right] \supset\left[\frac{x_{2}-1}{2^{2}}, \frac{x_{2}+1}{2^{2}}\right] \supset\left[\frac{x_{3}-1}{2^{3}}, \frac{x_{3}+1}{2^{3}}\right] \supset \cdots
$$

Formally, a real is defined as an infinite sequence $x$ of integers such that $\forall n\left|x_{n+1}-2 x_{n}\right| \leq 1$, with order relations

$$
\begin{aligned}
x<y & \Leftrightarrow \exists n x_{n}<y_{n}-2 \\
x \# y & \Leftrightarrow \exists n\left|x_{n}-y_{n}\right|>2 \\
x \leq y & \Leftrightarrow \forall n x_{n} \leq y_{n}+2 \\
x=y & \Leftrightarrow \forall n\left|x_{n}-y_{n}\right| \leq 2 .
\end{aligned}
$$

I call this the binary representation. Formally this represents $\mathbb{R}$ as a spread on integers. A spread on integers is a decidable predicate $s$ of finite sequences of integers such that $s(\langle\rangle)$ and $\forall u(s(u) \Leftrightarrow \exists z s(u *\langle z\rangle))$, where \langle\rangle is the empty sequence and $u *\langle z\rangle$ is the sequence $u$ extended by the integer $z$. An infinite sequence $x$ of integers is said to be a member of $s$ iff $\forall n s\left(\left\langle x_{0}, \ldots x_{n-1}\right\rangle\right)$. In the present case the spread $\mathbb{R}$ is defined by

$$
\mathbb{R}\left(\left\langle x_{0}, \ldots x_{n-1}\right\rangle\right) \Leftrightarrow \forall i<n-1\left|x_{i+1}-2 x_{i}\right| \leq 1
$$


Def ${ }^{\mathbf{n}}$. For any decidable property $P$ of natural numbers, the derivative $P^{\prime}$ of $P$ is the decidable property of natural numbers defined by

$$
\forall n\left(P^{\prime}(n) \Leftrightarrow P(n) \wedge \forall r<n \neg P(r)\right) .
$$

When I say that $P$ is 'decidable' I simply mean $\forall n(P(n) \vee \neg P(n))$. $P$ may be lawlike or may be defined by a sequence of free choices or a physical process such as coin-tossing, anything that gives a truth-value $P(n)$ for any desired $n$.

If $P^{\prime}(n)$ then Brouwer calls $n$ the critical number of $P$.

Theorem 1 For any decidable property $P$ of natural numbers,

(i) $\exists n P(n) \Leftrightarrow \exists n P^{\prime}(n)$

(ii) $\exists \leq 1 n P^{\prime}(n)$

(iii) $\neg \neg \exists n P^{\prime}(2 n) \Rightarrow \neg \exists n P^{\prime}(2 n+1)$ and $\neg \neg \exists n P^{\prime}(2 n+1) \Rightarrow \neg \exists n P^{\prime}(2 n)$.

\section{Axioms for the Creative Subject}

We choose an infinite increasing sequence of times, called stages; this sequence is called a schedule. $\vdash_{n} A$ means that the creative subject has a proof of $A$ at stage $n$; $\nvdash_{n} A$ means $\neg \vdash_{n} A$. I shall abbreviate $\exists n \vdash_{n} A$ to $\square A$.

Here are some possible axiom schemata, mostly dating back to Kreisel [29].

0. $\vdash_{n} A \vee \nvdash_{n} A$

1. $\square A \Rightarrow A$

$1^{\prime} . \quad \neg A \Rightarrow \neg \square A$

2. $A \Rightarrow \square A$

2'. $\neg \square A \Rightarrow \neg A$

3. $\neg(\square A \wedge \square \neg A)$

Kreisel himself adopted axioms 0, 1 and $2^{\prime}$ (and generalised the theory to allow for more than one creative subject). I have added axiom 3 for use alongside axiom 2 or $2^{\prime}$, for someone who does not accept axiom 1 or $1^{\prime}$. Two other axiom schemata are commonly proposed [30]:

$$
\begin{aligned}
& \vdash_{n} A \Rightarrow \vdash_{n+m} A \\
& \text { if } \vdash_{n} A \text { and } B \text { is an immediate consequence of } A \text { then } \vdash_{n} B
\end{aligned}
$$

I shall have no need for these.

Theorem 2 The following relationships exist between the axiom schemata.

(i) $1 \Rightarrow 1^{\prime} \Rightarrow 3$

(ii) $2 \Rightarrow 2^{\prime}$

(iii) $2^{\prime} \wedge 3 \Rightarrow 1^{\prime}$

I shall assume axiom 0 tacitly all the time; I shall explicitly mark all uses of the others in square brackets. 
Theorem 3 For any proposition A,

(i) $\square \neg A \Rightarrow \neg \square A$ and $\square A \Rightarrow \neg \square \neg A \quad$ [axiom 3]

(ii) $\neg \neg(\square A \vee \square \neg A) \quad$ [axiom $2^{\prime}$ ]

(iii) $\square \neg A \Rightarrow \neg A \quad$ [axiom $1^{\prime}$ ]

(iv) $\square A \vee \square \neg A \Rightarrow A \vee \neg A \quad$ [axiom 1]

(v) $\square A \vee \square \neg A \Rightarrow \neg \neg A \vee \neg A \quad$ [axiom $1^{\prime}$ ]

(vi) $\square A \vee \neg \square A \Rightarrow A \vee \neg A \quad$ [axioms $1,2^{\prime}$ ]

(vii) $\square A \vee \neg \square A \Rightarrow \neg \neg A \vee \neg A \quad$ [axioms 1', 2']

(viii) $\neg \square A \vee \neg \neg \square A \Rightarrow \neg A \vee \neg \neg A \quad$ [axioms $1^{\prime}, 2^{\prime}$ ]

(ix) $\neg \square A \vee \neg \square \neg A \Rightarrow \neg A \vee \neg \neg A \quad$ [axiom 2']

\section{The General form of a Weak Counterexample}

Brouwer stated his weak counterexamples by starting with a fleeing property or an unjudgeable or untestable proposition, assuming a hypothesis, and deriving a contradiction. I prefer to rephrase them by starting with any decidable property, or any proposition, assuming the hypothesis, and deriving the principle of excluded middle or something else constructively invalid.

Def $^{\text {n }}$. A weak counterexample (without creative subject) to a general proposition $\forall x B(x)$ is a theorem of the form

$$
\forall P \exists x(B(x) \Rightarrow I(P)),
$$

which implies

$$
\forall x B(x) \Rightarrow \forall P I(P),
$$

where $x$ may range over any set, $P$ ranges over all decidable properties of natural numbers, and $I(P)$ is a formula that is logically valid classically but not constructively, such as

(i) $\exists n P(n) \vee \neg \exists n P(n)$,

(ii) $\neg \exists n P(n) \vee \neg \neg \exists n P(n)$,

(iii) $\neg \neg \exists n P(n) \Rightarrow \exists n P(n)$,

(iv) $\neg \exists n P^{\prime}(2 n) \vee \neg \exists n P^{\prime}(2 n+1)$,

(v) $\neg \neg \exists n P(n) \Rightarrow \neg \exists n P^{\prime}(2 n) \vee \neg \exists n P^{\prime}(2 n+1)$.

This way of formulating weak counterexamples follows Bridges \& Richman [2, $\S 1.1]$ and Troelstra \& van Dalen [38, §1.3.5]; the schema (i) as known as the limited principle of omniscience, (ii) is the weak limited principle of omniscience, (iii) is Markov's principle, (iv) is essentially the lesser limited principle of omniscience; the schemata (iv) and (v) have equivalent versions,

(iv) $\neg(\exists n P(n) \wedge \exists n Q(n)) \Rightarrow \neg \exists n P(n) \vee \neg \exists n Q(n)$,

(v) $\neg \neg(\exists n P(n) \vee \exists n Q(n)) \wedge \neg(\exists n P(n) \wedge \exists n Q(n)) \Rightarrow \neg \exists n P(n) \vee$ $\neg \exists n Q(n)$,

for decidable $P, Q$. The logical relations between these schemata are: (i) $\Rightarrow$ (ii) $\Rightarrow$ (iv) $\Rightarrow$ (v) and (i) $\Rightarrow$ (iii) $\Rightarrow$ (v). Constructivists of the 'Russian' school [2] accept 
Markov's principle as valid, but only for recursive $P$, not for all $P$ decidable in the very broad sense I am using here. Schemata (i), (ii) and (iv) are considered invalid by all constructivists.

In weak counterexamples in [41, p. 42, pp. 55-6] Brouwer uses a generalisation of (v), in which even and odd numbers are replaced by two arbitrary decidable complementary subspecies of $\mathbb{N}$, called $\beta(A)$ and $\gamma(A)$; but this generalisation does not seem fruitful, and I shall stick to even and odd numbers.

In some cases the converse, $\forall P I(P) \Rightarrow \forall x B(x)$, also holds, though Brouwer did not discuss this.

Occasionally a stronger form of weak counterexample appears, $\forall P \forall x(B(x) \Rightarrow$ $I(P))$ or $\exists x B(x) \Rightarrow \forall P I(P)$; see theorems 15a and 19a below. This is used to show the non-existence of an $x$ such that $B(x)$.

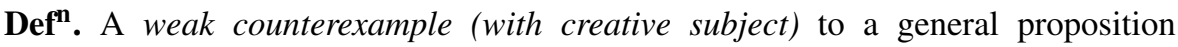
$\forall x B(x)$ is a theorem of the form

$$
\forall A \exists x(B(x) \Rightarrow I(A)),
$$

which implies

$$
\forall x B(x) \Rightarrow \forall A I(A),
$$

where $A$ ranges over all propositions and $I(A)$ is a formula that is logically valid classically but not constructively, such as

$$
\begin{aligned}
& A \vee \neg A, \\
& \neg A \vee \neg \neg A, \\
& \neg \neg A \Rightarrow A .
\end{aligned}
$$

Every weak counterexample with the creative subject can be derived from a corresponding version without the creative subject, using the following terminology.

Def" . Given any proposition $A$, define three decidable properties of natural numbers as follows:

(i) the conditional property $P$ of $A$ is defined by

$$
\forall n\left(P(n) \Leftrightarrow \vdash_{n} A\right) ;
$$

(ii) the direct property $P$ of $A$ is defined by

$$
\forall n\left(P(n) \Leftrightarrow \vdash_{n} A \vee \vdash_{n} \neg A\right) ;
$$

(iii) the oscillatory property $P$ of $A$ is defined by

$$
\begin{array}{cl}
\forall n(P(2 n) & \left.\Leftrightarrow \vdash_{n} A\right) \\
\forall n(P(2 n+1) & \left.\Leftrightarrow \vdash_{n} \neg A\right) .
\end{array}
$$

It is immediate that, for any proposition $A$, having conditional property $P$, we have $\exists n P(n) \Leftrightarrow \square A$.

Theorem 4 For any proposition A, having direct property $P$,

(i) $\exists n P(n) \Leftrightarrow \square A \vee \square \neg A$

(ii) $\neg \neg \exists n P(n) . \quad$ [axiom $\left.2^{\prime}\right]$ 
Theorem 5 For any proposition A, having oscillatory property $P$,

(i) $\exists n P(n) \Leftrightarrow \square A \vee \square \neg A$

(ii) $\neg \neg \exists n P(n) \quad$ [axiom $\left.2^{\prime}\right]$

(iii) $\square A \Rightarrow \exists n P^{\prime}(2 n)$ [axiom 3], and $\exists n P^{\prime}(2 n) \Rightarrow \square A$

(iv) $\square \neg A \Rightarrow \exists n P^{\prime}(2 n+1)$ [axiom 3], and $\exists n P^{\prime}(2 n+1) \Rightarrow \square \neg A$.

\section{The Weak Counterexamples}

I shall give each weak counterexample in two versions; e.g., theorem 7a is the version without the creative subject and theorem $7 \mathrm{~b}$ is the version with the creative subject. (Theorem 11 has three versions.)

I shall just give the constructions and brief details of proofs where they are interesting. Proofs will be written in a natural deduction style, using indented lines to indicate the scope of hypotheses or universal quantifiers. Indentation increases after a hypothesis or universal quantifier is introduced, and decreases when it is discharged. Here is a simple example of the format:

Consider any $x \in \mathbb{R}$.

$$
\begin{aligned}
& \text { Then } x^{2}-4 x+4=(x-2)^{2} \geq 0, \\
& \text { so } x^{2}+4 \geq 4 x
\end{aligned}
$$

This shows that $\forall x \in \mathbb{R} x^{2}+4 \geq 4 x$.

\subsection{Weak Counterexamples Involving Real Numbers}

Theorem 6a (binary approximation number). For any decidable property $P$ of natural numbers, there exists a unique $x \in \mathbb{R}$ such that

(i) $x \# 0 \Rightarrow \exists n P(n)$

(ii) $\forall n\left(P^{\prime}(n) \Rightarrow x=2^{-n}\right)$;

and this $x$ also satisfies

(iii) $x \geq 0$

(iv) $x>0 \Leftrightarrow \exists n x=2^{-n} \Leftrightarrow \exists n P(n)$

(v) $x=0 \Leftrightarrow \neg \exists n P(n)$

(vi) $x \in \mathbb{Q} \Leftrightarrow x \# 0 \vee x=0 \Leftrightarrow x>0 \vee x \leq 0 \Leftrightarrow \exists n P(n) \vee \neg \exists n P(n)$

(vii) $x \notin \mathbb{Q}$

(viii) $x=0 \vee x \neq 0 \Leftrightarrow x=0 \vee x \# 0 \Leftrightarrow x \leq 0 \vee x \not \leq 0 \Leftrightarrow \neg \exists n P(n) \vee$ $\neg \neg \exists n P(n)$.

(Brouwer uses parts (iii,viii) to refute $\forall x(x \geq 0 \Rightarrow x=0 \vee x \not 0)$ [41, p. 41]. Conversely, the schema $\exists n P(n) \vee \neg \exists n P(n)$ immediately implies $\forall x(x \# 0 \vee$ $x=0)$ and $\forall x(x>0 \vee x \leq 0)$; the schema $\neg \exists n P(n) \vee \neg \neg \exists n P(n)$ implies $\forall x(x=0 \vee x \neq 0)$ and $\forall x(x \leq 0 \vee x \not \leq 0)$. $)$

Proof Consider any decidable property $P$ of natural numbers. 
Brouwer gives two equivalent constructions for $x$.

(Construction 1 [41, p. 41])

Define a rational sequence $a$ by

$$
\forall n \quad a_{n}= \begin{cases}0 & \text { if } \forall i \leq n \neg P(i) \\ 2^{-r} & \text { if } r \leq n \text { and } P^{\prime}(r)\end{cases}
$$

This is a Cauchy sequence, so define $x=\lim _{n \rightarrow \infty} a_{n}$.

(Construction 2 [10, p. 426], [12, pp. 444-5])

Define a rational sequence $b$ by

$$
\forall n \quad b_{n}= \begin{cases}2^{-n} & \text { if } \forall i \leq n \neg P(i) \\ 2^{-r} & \text { if } r \leq n \text { and } P^{\prime}(r)\end{cases}
$$

This is a Cauchy sequence, so define $x=\lim _{n \rightarrow \infty} b_{n}$.

All the parts of the theorem are easily verified.

Theorem 6b. For any proposition A, there exists $x \in \mathbb{R}$ such that

(i) $x \geq 0$

(ii) $x>0 \Leftrightarrow \exists n x=2^{-n} \Leftrightarrow \square A \vee \square \neg A \Rightarrow \begin{cases}A \vee \neg A & \text { [axiom 1] } \\ \neg \neg A \vee \neg A & \text { [axiom 1'] }\end{cases}$

(iii) $x \not 0 \quad\left[\right.$ axiom $\left.2^{\prime}\right]$

(iv) $x \in \mathbb{Q} \Leftrightarrow x \# 0 \Leftrightarrow x>0 \quad$ [axiom $2^{\prime}$ ]

(v) $x \notin \mathbb{Q}$.

(Brouwer uses parts (ii,iii) to show that $x \not 0$ does not imply $x>0$ [13] [41, p. 50]. He seems to be assuming axiom $1^{\prime}$ rather than 1 , since he considers an 'untestable assertion' rather than an 'unjudgeable assertion'.)

Proof For any proposition $A$, construct its direct property $P$ and apply theorem 6 a. The theorem then follows with the help of theorem 3 and theorem 4 .

Theorem 7a (dual pendulum number, oscillatory binary shrinking number). For any decidable property $P$ of natural numbers, there exists a unique $x \in \mathbb{R}$ such that

(i) $x \# 0 \Rightarrow \exists n P(n)$

(ii) $\forall n\left(P^{\prime}(n) \Rightarrow x=(-2)^{-n}\right)$;

and this $x$ also satisfies

(iii) $x>0 \Leftrightarrow \exists n P^{\prime}(2 n)$

(iv) $x<0 \Leftrightarrow \exists n P^{\prime}(2 n+1)$

(v) $x \# 0 \Leftrightarrow \exists n x=(-2)^{-n} \Leftrightarrow \exists n P(n)$

(vi) $x \not 0 \vee x \leq 0 \Leftrightarrow \neg \neg \exists n P^{\prime}(2 n) \vee \neg \exists n P^{\prime}(2 n)$

(vii) $x \in \mathbb{Q} \Leftrightarrow x \# 0 \vee x=0 \Leftrightarrow \exists n P(n) \vee \neg \exists n P(n)$

(viii) $x \notin \mathbb{Q}$ 
(ix) $\quad x=0 \vee x \neq 0 \Leftrightarrow \neg \exists n P(n) \vee \neg \neg \exists n P(n)$

(x) $x=0 \vee x \not 0 \vee x \not 0 \Rightarrow x \leq 0 \vee x \geq 0 \Leftrightarrow \neg \exists n P^{\prime}(2 n) \vee$ $\neg \exists n P^{\prime}(2 n+1)$

(xi) $(x \neq 0 \Rightarrow x \notin 0 \vee x \not 0) \Rightarrow\left(\neg \neg \exists n P(n) \Rightarrow \neg \exists n P^{\prime}(2 n) \vee\right.$ $\left.\neg \exists n P^{\prime}(2 n+1)\right)$.

\section{(Brouwer uses}

- $\quad$ part (vi) to refute $\forall x(x \# 0 \vee x \leq 0)$ and $\forall x((x \# 0 \vee x \leq 0) \vee \neg(x \#$ $0 \vee x \leq 0))[10$, p. 425];

- $\quad$ parts (vii,viii) to refute $\forall x(x \notin \mathbb{Q} \Rightarrow x \in \mathbb{Q})[41$, pp.6-7] and $\forall x(x \in \mathbb{Q} \vee$ $x \notin \mathbb{Q}$ ) [10, p. 425] [12, p. 444], and to show that $x \notin \mathbb{Q}$ does not imply $x \in \mathbb{Q} \vee$ $x \notin \mathbb{Q}[25]$; by taking $A$ and $B$ both as $x \in \mathbb{Q}$, this refutes $\neg(\neg A \vee \neg B) \Leftrightarrow$ $A \wedge B$ and $A \vee B \Leftrightarrow \neg(\neg A \wedge \neg B)$ [25];

- $\quad$ part (ix) to refute $\forall x(x=0 \vee x \neq 0)$ [10, p. 425] [11, p. 59] [12, p. 444] [41, pp. 6-7], and to show that $\neg \neg x=0 \Rightarrow x=0$ (which always holds) does not imply $x=0 \vee x \neq 0[25]$;

- $\operatorname{part}(\mathrm{x})$ to refute $\forall x(x=0 \vee x \sharp 0 \vee x \# 0)$ [12, p. 444] [41, p. 41] and $\forall x(x \leq 0 \vee x \geq 0)[41$, pp. 6-7];

- $\quad$ part (xi) to refute $\forall x(x \neq 0 \Rightarrow x \notin 0 \vee x \# 0)$ [41, p. 42]; by taking $A$ as $x \geq 0$ and $B$ as $x \leq 0$, this refutes $\neg(A \wedge B) \Leftrightarrow \neg A \vee \neg B$ [25].

He also uses this in other counterexamples [12]; see theorem 20a and theorem 24a below. This construction is used together with the binary approximation number in geometric counterexamples [10, p. 426]. Conversely, the schema $\neg \exists n P^{\prime}(2 n) \vee$ $\neg \exists n P^{\prime}(2 n+1)$ implies $\forall x(x \leq 0 \vee x \geq 0)$; the schema $\neg \neg \exists n P(n) \Rightarrow$ $\neg \exists n P^{\prime}(2 n) \vee \neg \exists n P^{\prime}(2 n+1)$ implies $\forall x(x \neq 0 \Rightarrow x \notin 0 \vee x \sharp 0)$.)

Proof Consider any decidable property $P$ of natural numbers.

Brouwer gives two equivalent constructions for $x$.

(Construction 1 [41, p. 41])

Define a rational sequence $a$ by

$$
\forall n \quad a_{n}= \begin{cases}0 & \text { if } \forall i \leq n \neg P(i) \\ (-2)^{-r} & \text { if } r \leq n \text { and } P^{\prime}(r)\end{cases}
$$

This is a Cauchy sequence, so define $x=\lim _{n \rightarrow \infty} a_{n}$.

(Construction 2 [5, 6], [10, p. 425], [12, p. 444], [25], [41, pp. 6-7])

Define a rational sequence $b$ by

$$
\forall n \quad b_{n}= \begin{cases}(-2)^{-n} & \text { if } \forall i \leq n \neg P(i) \\ (-2)^{-r} & \text { if } r \leq n \text { and } P^{\prime}(r)\end{cases}
$$

This is a Cauchy sequence, so define $x=\lim _{n \rightarrow \infty} b_{n}$.

All parts of the theorem are easily verified, with the help of theorem 1(iii). 
Theorem 7b. For any proposition $A$, there exists $x \in \mathbb{R}$ such that

(i) $x>0 \Leftrightarrow \square A \quad$ [axiom 3]

(ii) $x<0 \Leftrightarrow \square \neg A \quad$ [axiom 3]

(iii) $x \neq 0 \quad\left[\right.$ axiom $\left.2^{\prime}\right]$

(iv) $x \in \mathbb{Q} \Leftrightarrow x \# 0 \Leftrightarrow \square A \vee \square \neg A \Rightarrow \begin{cases}A \vee \neg A & \text { [axioms } 1,2^{\prime} \text { ] } \\ \neg \neg A \vee \neg A & \text { [axioms } 1^{\prime}, 2^{\prime} \text { ] }\end{cases}$

(v) $x \notin \mathbb{Q}$

(vi) $x \not 0 \vee x \# 0 \Leftrightarrow x \leq 0 \vee x \geq 0 \Leftrightarrow \neg \square A \vee \neg \square \neg A \Rightarrow \neg A \vee \neg \neg A$ [axioms $\left.2^{\prime}, 3\right]$

(vii) $\quad x \notin 0 \vee x \# 0 \Rightarrow \neg \neg \square \neg A \vee \neg \neg \square A \Rightarrow \neg A \vee \neg \neg A . \quad$ [axiom 1']

(Brouwer uses parts (iii,vi) to show that

- $\quad$ \# is not a simple order [42] [11, p. 59] [13] [41, p. 43];

- consequently $x \# 0$ is not equivalent to $x \neq 0$ [13].

Part (vii) is an alternative argument to (vi), not used by Brouwer - see discussion below in $\$ 7.2$.

Proof For any proposition $A$, construct its oscillatory property $P$ and apply theorem 7a. The theorem then follows with the help of theorem 3 and theorem 5.

Note: if $x$ is the oscillatory binary shrinking number then $|x|$ is the binary approximation number.

Theorem 8a. For any decidable property $P$ of natural numbers, there exist $x, y \in \mathbb{R}$ such that

(i) $0 \leq x \leq y$

(ii) $0<y \Leftrightarrow \exists n P(n)$

(iii) $0<x \Leftrightarrow \exists n P^{\prime}(2 n)$

(iv) $x<y \Leftrightarrow \exists n P^{\prime}(2 n+1)$

(v) $(0 \not 4 y \Rightarrow 0 \not x \vee x \notin y) \Rightarrow\left(\neg \neg \exists n P(n) \Rightarrow \neg \exists n P^{\prime}(2 n+1) \vee\right.$ $\left.\neg \exists n P^{\prime}(2 n)\right)$.

(This is a weak counterexample to $\forall x, y(0 \sharp y \Rightarrow 0 \sharp x \vee x \sharp y)$.)

Proof

Consider any decidable property $P$ of natural numbers.

Choose $u$ as the oscillatory binary shrinking number (theorem 7a);

choose $x \equiv \max (0, u)$ and $y \equiv|u|=\max (-u, u)$.

Then parts (i-v) follow.

Theorem 8b. For any proposition A, there exist $x, y \in \mathbb{R}$ such that

(i) $0 \leq x \leq y$

(ii) $0<x \Rightarrow \square A$

(iii) $x<y \Rightarrow \square \neg A$ 
(iv) $0 \not y \quad\left[\right.$ axiom $\left.2^{\prime}\right]$

(v) $0 \# x \vee x \sharp y \Rightarrow \neg \neg \square A \vee \neg \neg \square \neg A \Rightarrow \neg \neg A \vee \neg A$. [axiom $1^{\prime}$ ]

(This is a strengthened version of an example by Heyting [27, §8.1.1].)

Proof For any proposition $A$, construct the oscillatory property $P$ and apply theorem 8a. The theorem then follows with the help of theorem 3 and theorem 5.

We now generalise by replacing powers of 2 and -2 by arbitrary convergent sequences of reals.

Def $^{\mathbf{n}}$. A drift is a pair $(a, u)$, where $a$ is a real number and $u$ is a sequence of real numbers such that $u_{n} \rightarrow a$ as $n \rightarrow \infty$.

(This is defined in [14]. Brouwer imposes the extra conditions that $\forall n u_{n} \# a$ and $\forall m, n\left(m \neq n \Rightarrow u_{m} \# u_{n}\right)$; but I shall only assume these conditions when necessary. The latter condition seems unnecessarily strong.)

The following theorem uses the binary representation of real numbers (see §2).

Theorem 9 For any drift $(a, u)$, there exist a function $v: \mathbb{N} \rightarrow \mathbb{N}$, a real number $a^{\prime}$, and a sequence $u^{\prime}$ of real numbers, such that

(i) $\forall n v(n+1) \geq v(n)$ and $\forall n v(n) \geq n$

(ii) $a^{\prime}=a$ and $\forall r u_{r}^{\prime}=u_{r}$

(iii) $\forall r, n\left(v(n)<r \Rightarrow\left|u_{r, n+1}^{\prime}-2 a_{n}^{\prime}\right| \leq 1\right)$.

(Notation: $u^{\prime}$ is a sequence of real numbers; $u_{r}^{\prime}$ is the $r$ th real number in the sequence; a real number is itself a sequence of integers, so $u_{r, n+1}^{\prime}$ is the $(n+1)$ th integer of $u_{r}^{\prime}$.)

Proof

Consider any drift $(a, u)$.

Choose a function $N: \mathbb{N} \rightarrow \mathbb{N}$ such that $\forall i \forall r>N(i)\left|u_{r}-a\right| \leq 2^{-i}$.

Define a function $v: \mathbb{N} \rightarrow \mathbb{N}$ by

$$
\begin{aligned}
& =N(4) \\
\forall n v(n+1) & =\max (v(n), N(n+5), n+1) .
\end{aligned}
$$

Then part (i) follows.

(ii) Define $a^{\prime} \in \mathbb{R}$, and, for every $r, u_{r}^{\prime} \in \mathbb{R}$ by

$$
\begin{array}{r}
\forall n a_{n}^{\prime}=\operatorname{round}\left(\frac{a_{n+4}}{16}\right) \\
\forall r, n u_{r n}^{\prime}=\operatorname{round}\left(\frac{u_{r, n+3}}{8}\right)
\end{array}
$$

where round rounds a rational number to the nearest integer, rounding halves arbitrarily. 
Then $a^{\prime}=a$ and $\forall r u_{r}^{\prime}=u_{r}$, as required.

(iii) can then be verified.

This proves the theorem for any $\operatorname{drift}(a, u)$.

Def ${ }^{\text {n }}$ A checking-number of a drift $(a, u)$ through a decidable property $P$ of natural numbers is a real number $x$ such that

(a) $x \# a \Rightarrow \exists n P(n)$

(b) $\forall n\left(P^{\prime}(n) \Rightarrow x=u_{n}\right)$.

Theorem 10a. For any drift $(a, u)$ and any decidable property $P$ of natural numbers, there exists a unique checking-number $x$ of $(a, u)$ through $P$, and $x$ satisfies

(i) $\neg \neg\left(x=a \vee \exists n x=u_{n}\right)$

(ii) if $\forall n u_{n} \# a$ then $x \# a \Leftrightarrow \exists n x=u_{n} \Leftrightarrow \exists n P(n)$

(iii) if $\forall n u_{n} \# a$ and $\forall m, n\left(u_{m}=u_{n} \Rightarrow m=n\right)$ then $\forall n\left(P^{\prime}(n) \Leftrightarrow x=u_{n}\right)$;

if $\forall n a<u_{n}$ then

(iv) $a \leq x$

(v) $a<x \Leftrightarrow \exists n u_{n}<x \Leftrightarrow \exists n u_{n} \not x \Leftrightarrow \exists n P(n)$

(vi) $a \not x \Leftrightarrow \neg \neg \exists n P(n)$.

(Hence the schema $\neg \neg \exists n P(n) \Rightarrow \exists n P(n)$ is equivalent to $\forall x$ (a $\nleftarrow x \Rightarrow a<$ $x)$.)

Proof

Consider any drift $(a, u)$ and any decidable property $P$ of natural numbers.

I shall give two equivalent constructions of a checking-number $x$.

(Construction 1, using the binary representation of real numbers (cf theorem 30 below))

Define a sequence $x$ of integers by

$$
\forall n \quad x_{n}= \begin{cases}u_{r n}^{\prime} & \text { if } r \leq v(n) \text { and } P^{\prime}(r) \\ a_{n}^{\prime} & \text { if } \forall i \leq v(n) \neg P(i)\end{cases}
$$

using $v, a^{\prime}, u^{\prime}$ as supplied by theorem 9 .

Then it follows that $x \in \mathbb{R}$, and conditions (a) and (b) follow.

(Construction 2, not using any representation of real numbers)

Define a sequence $s$ of real numbers by

$$
\forall n s_{n}= \begin{cases}u_{r} & \text { if } r \leq n \text { and } P^{\prime}(r) \\ a & \text { if } \forall i \leq n \neg P(i) .\end{cases}
$$

Then $s$ is a Cauchy sequence, so define $x=\lim _{n \rightarrow \infty} s_{n}$.

Conditions (a) and (b) can be verified.

Uniqueness of the checking-number and parts (i-vi) follow. 
Def ${ }^{\mathbf{n}}$. The conditional checking-number of a drift $(a, u)$ through a proposition $A$ is the checking-number of $(a, u)$ through the conditional property of $A$. [15]

The direct checking-number of a drift $(a, u)$ through a proposition $A$ is the checking-number of $(a, u)$ through the direct property of $A$. [14]

The oscillatory checking-number of a drift $(a, u)$ through a proposition $A$ is the checking-number of $(a, u)$ through the oscillatory property of $A$. [14]

Each of these three kinds of checking-number is given by an infinite sequence whose $n$th term is determined at stage $v(n)$ or stage $n$. Therefore these checkingnumbers are dependent on the timing of the stages relative to the timing of the creative subject's constructive activity that may lead to a proof of $A$ or $\neg A$. Let us assume for now that these timings are fixed; then the conditional, direct or oscillatory checkingnumber of a given drift through a given proposition is uniquely determined. (I shall return to this question of timings in §9.1.)

Theorem 10b. For any drift $(a, u)$ and any proposition A, with direct checkingnumber $x \in \mathbb{R}$,

(i) $\neg \neg\left(x=a \vee \exists n x=u_{n}\right)$;

if $\forall n u_{n} \#$ a then

(ii) $x \# a \Leftrightarrow \exists n x=u_{n} \Leftrightarrow \square A \vee \square \neg A$

(iii) $x \neq a ; \quad$ [axiom $2^{\prime}$ ]

if $\forall n u_{n} \# a$ and $\forall m, n\left(u_{m}=u_{n} \Rightarrow m=n\right)$ then

(iv) $\forall n\left(\left(\vdash_{n} A \vee \vdash_{n} \neg A\right) \wedge \forall i<n\left(\nvdash_{i} A \wedge \nvdash_{i} \neg A\right) \Leftrightarrow x=u_{n}\right)$;

if $\forall n a<u_{n}$ then

(v) $a \sharp x \quad$ [axiom 2']

(vi) $a<x \Leftrightarrow \exists n u_{n}<x \Leftrightarrow \exists n u_{n} \not \quad x \Leftrightarrow \square A \vee \square \neg A \Rightarrow$ $\left\{\begin{array}{l}A \vee \neg A \quad[\text { axiom } 1] \\ \neg \neg A \vee \neg A .[\text { axiom } 1 \text { '] }\end{array}\right.$

(Brouwer uses parts (v,vi)

- to show that $a \nVdash x$ does not imply $a<x$ [14, p. 492]; he seems to assume axiom $1^{\prime}$;

- to show that $a \nVdash x$ does not imply $\exists n u_{n} \sharp x[11, \mathrm{p} .60]$.)

Proof For any drift $(a, u)$ and any proposition $A$, construct the direct property of $A$ and apply theorem 10a. The theorem then follows.

Theorem 11a. For any drift $(a, u)$ such that $\forall n u_{n} \in \mathbb{Q}$, and any decidable property $P$ of natural numbers, with checking-number $x \in \mathbb{R}$,

(i) $\exists n P(n) \Rightarrow x \in \mathbb{Q}$;

if $a \notin \mathbb{Q}$ then

(ii) $x \notin \mathbb{Q} \Leftrightarrow \neg \exists n P(n)$

(iii) $x \notin \mathbb{Q} \vee x \notin \mathbb{Q} \Leftrightarrow \neg \exists n P(n) \vee \neg \neg \exists n P(n)$;

if $\forall q \in \mathbb{Q} a \# q$ then

(iv) $x \in \mathbb{Q} \Leftrightarrow \exists n P(n)$

(v) $x \in \mathbb{Q} \vee x \notin \mathbb{Q} \Leftrightarrow \exists n P(n) \vee \neg \exists n P(n)$. 
(This construction

- $\quad$ is a weak counterexample to $x \notin \mathbb{Q} \vee x \notin \mathbb{Q}$,

- $\quad$ shows that $x \notin \mathbb{Q}$ does not imply $x \in \mathbb{Q}$, and so $x \notin \mathbb{Q} \vee x \notin \mathbb{Q}$ does not imply $x \in \mathbb{Q} \vee x \notin \mathbb{Q}$.)

Proof This follows easily from the properties of a checking-number.

Theorem 11b. For any drift $(a, u)$ such that $\forall n u_{n} \in \mathbb{Q}$, and any proposition $A$, with conditional checking-number $x \in \mathbb{R}$,

(i) $A \Rightarrow x \in \mathbb{Q} ; \quad$ [axiom 2]

if $a \notin \mathbb{Q}$ then

(ii) $x \notin \mathbb{Q} \Leftrightarrow \neg A \quad$ [axioms $1^{\prime}, 2^{\prime}$ ]

(iii) $x \notin \mathbb{Q} \vee x \notin \mathbb{Q} \Leftrightarrow \neg A \vee \neg \neg A ; \quad$ [axioms $1^{\prime}, 2^{\prime}$ ]

if $\forall q \in \mathbb{Q} a \# q$ then

(iv) $x \in \mathbb{Q} \Leftrightarrow A \quad$ [axioms 1,2]

(v) $\quad x \in \mathbb{Q} \vee x \notin \mathbb{Q} \Leftrightarrow A \vee \neg A$. [axioms 1,2]

(Brouwer uses this as a counterexample to $x \notin \mathbb{Q} \vee x \notin \mathbb{Q}[20$, p. 525]. He also makes the stronger claim that $a \notin \mathbb{Q}$ implies $x \in \mathbb{Q} \Leftrightarrow A$, which I consider unsound; see $\$ 9.1$ below. Since he says that $A$ is untestable, rather than unjudgeable, he seems to be using axiom $1^{\prime}$ rather than 1.)

Proof For any such drift $(a, u)$ and any proposition $A$, construct the conditional property of $A$ and apply theorem 11a. The theorem then follows.

Theorem 11c. For any drift $(a, u)$ such that $\forall n u_{n} \in \mathbb{Q}$, and any proposition $A$, with direct checking-number $x \in \mathbb{R}$,

(i) $A \vee \neg A \Rightarrow x \in \mathbb{Q} ; \quad$ [axiom 2]

(ii) $x \notin \mathbb{Q} ; \quad\left[\right.$ axiom $\left.2^{\prime}\right]$

if $\forall q \in \mathbb{Q} a \# q$ then

(iii) $x \in \mathbb{Q} \Rightarrow \begin{cases}A \vee \neg A & {[\text { axiom } 1]} \\ \neg \neg A \vee \neg A . & {[\text { axiom } 1 ']}\end{cases}$

(Brouwer claims that $x \notin \mathbb{Q}$ is not equivalent to $x \in \mathbb{Q}$ and that we have $x \notin \mathbb{Q} \vee$ $x \notin \mathbb{Q}$ but cannot prove $x \in \mathbb{Q} \vee x \notin \mathbb{Q}$ [14, p. 492]; see also [20, p. 525], where he assumes $a \notin \mathbb{Q}$; the claims are unjustified because he has not assumed $\forall q \in \mathbb{Q} a \# q$. See $\$ 9.1$ for discussion.)

Proof For any such drift $(a, u)$ and any proposition $A$, construct the direct property of $A$ and apply theorem 11a. The theorem then follows.

Theorem 12a. For any drift $(a, u)$ such that $a \in \mathbb{Q}$ and $\forall n u_{n} \notin \mathbb{Q}$, and any decidable property $P$ of natural numbers, with checking-number $x \in \mathbb{R}$,

(i) $\neg \neg \exists n P(n) \Rightarrow x \notin \mathbb{Q}$ 
(ii) $\forall q \in \mathbb{Q} x \# q \Rightarrow \exists n P(n)$

(iii) $(x \notin \mathbb{Q} \Rightarrow \forall q \in \mathbb{Q} x \# q) \Rightarrow(\neg \neg \exists n P(n) \Rightarrow \exists n P(n))$.

Proof This follows easily from the properties of a checking-number.

Theorem 12b. For any drift $(a, u)$ such that $a \in \mathbb{Q}$ and $\forall n u_{n} \notin \mathbb{Q}$, and any proposition A, with direct checking-number $x \in \mathbb{R}$,

(i) $x \notin \mathbb{Q} \quad[$ axiom 2']

(ii) $\forall q \in \mathbb{Q} x \# q \Rightarrow \square A \vee \square \neg A \Rightarrow\left\{\begin{array}{l}A \vee \neg A \quad \text { [axiom 1] } \\ \neg \neg A \vee \neg A \text {. [axiom 1'] }\end{array}\right.$

(This is an example in [14, p. 494]. Brouwer uses it to show that $x \notin \mathbb{Q}$ does not imply $\forall q \in \mathbb{Q} x \# q$. Since he says that $A$ is untestable, rather than unjudgeable, he seems to be using axiom $1^{\prime}$ rather than 1.)

Proof For any such drift $(a, u)$ and proposition $A$, construct the direct property of $A$ and apply theorem $12 \mathrm{a}$. The theorem then follows.

Theorem 13a. For any drift $(a, u)$ such that such that $\forall n\left(u_{2 n} \in \mathbb{Q} \wedge u_{2 n}>a\right)$ and $\forall n\left(u_{2 n+1} \notin \mathbb{Q} \wedge u_{2 n+1}<a\right)$, and any decidable property $P$ of natural numbers, with checking-number $x \in \mathbb{R}$,

(i) $\exists n P^{\prime}(2 n) \Rightarrow x \in \mathbb{Q} \wedge x>a$

(ii) $\exists n P^{\prime}(2 n+1) \Rightarrow x \notin \mathbb{Q} \wedge x<a$

(iii) $x \notin \mathbb{Q} \vee x \notin \mathbb{Q} \Rightarrow \neg \exists n P^{\prime}(2 n) \vee \neg \exists n P^{\prime}(2 n+1)$

(iv) $x \leq a \vee x \geq a \Rightarrow \neg \exists n P^{\prime}(2 n) \vee \neg \exists n P^{\prime}(2 n+1)$.

(Conversely, the schema $\neg \exists n P^{\prime}(2 n) \vee \neg \exists n P^{\prime}(2 n+1)$ implies $\forall x(x \leq a \vee$ $x \geq a)$.)

Proof This follows easily from the properties of a checking-number.

Theorem 13b. For any drift $(a, u)$ such that $\forall n\left(u_{2 n} \in \mathbb{Q} \wedge u_{2 n}>a\right)$ and $\forall n\left(u_{2 n+1} \notin \mathbb{Q} \wedge u_{2 n+1}<a\right)$, and any proposition $A$, with oscillatory checkingnumber $x \in \mathbb{R}$,

(i) $\square A \Rightarrow x \in \mathbb{Q} \wedge x>a \quad$ [axiom 3]

(ii) $\square \neg A \Rightarrow x \notin \mathbb{Q} \wedge x<a \quad$ [axiom 3]

(iii) $x \notin \mathbb{Q} \vee x \notin \mathbb{Q} \Rightarrow \neg A \vee \neg \neg A \quad$ [axioms $\left.2^{\prime}, 3\right]$

(iv) $x \leq a \vee x \geq a \Rightarrow \neg A \vee \neg \neg A$. [axioms 2',3]

(This is an example in [14, p. 492]. Brouwer uses it

- $\quad$ as a counterexample to $x \notin \mathbb{Q} \vee x \notin \mathbb{Q}$ (and hence also to $x \in \mathbb{Q} \vee x \notin \mathbb{Q}$ ),

- as a counterexample to $x \geq a \vee x \leq a$.

Brouwer also claims that it shows that $x \notin \mathbb{Q}$ is not equivalent to $x \in \mathbb{Q}$, which is unjustified; see $\$ 9.1$ for discussion.) 
Proof For any such drift $(a, u)$ and proposition $A$, construct the oscillatory property of $A$ and apply theorem 13a. The theorem then follows.

\subsection{Weak Counterexamples Involving Orderings in General}

From now on I shall not state the 'b' forms of the theorems in full, as they can derived in a completely stereotyped way from the 'a' forms.

Def ${ }^{\text {n }}$ A partially ordered set is a triple $(A, \equiv, \prec)$, where $A$ is a set and $\equiv, \prec$ are binary relations on $A$, such that, for all $a, b, c, r, s \in A$,

(1) $a \equiv a$

(2) $a \equiv b \Rightarrow b \equiv a$

(3) $a \equiv b \wedge b \equiv c \Rightarrow a \equiv c$

(5) $a \equiv r \wedge b \equiv s \wedge a \prec b \Rightarrow r \prec s$

(6) $a \prec b \wedge b \prec c \Rightarrow a \prec c$

(7) $a \prec b \Rightarrow b \nprec a$.

(I omit Brouwer's axiom (4), $a \succ b \Leftrightarrow b \prec a$. I use $\not \equiv, \nprec$ for the negated relations.)

Def $^{\text {n }}$. A virtually ordered set is a partially ordered set $(A, \equiv, \prec)$ such that, for all $a, b \in A$,

(8) $a \not \equiv b \wedge a \nprec b \Rightarrow b \prec a$

(9) $a \nprec b \wedge b \nprec a \Rightarrow a \equiv b$.

Def ${ }^{\text {n }}$ A simply ordered (or quasi-completely ordered) set is a partially ordered set $(A, \equiv, \prec)$ such that, for all $a, b \in A$,

(10) $a \not \equiv b \Rightarrow a \prec b \vee b \prec a$.

\section{Theorem 14a.}

(i) For any decidable property $P$ of natural numbers, there is a partially ordered set that is a virtually ordered set iff $\neg \neg \exists n P(n) \Rightarrow \exists n P(n)$.

(ii) For any proposition $A$, there is a virtually ordered set that is a simply ordered set iff $\neg A \vee \neg \neg A$.

(iii) For any proposition $A$, there is a simply ordered set that is a virtually ordered set iff $\neg \neg A \Rightarrow A$.

(These three examples are from [16]. In (i) I have used decidable properties of natural numbers; Brouwer probably intended a use of the creative subject. In (ii,iii) I have interpreted Brouwer's expression 'l'ensemble de $X$ et $Y$ ' as $X \wedge Y$ rather than $X \vee Y$; this is necessary to make the argument work. In English he sometimes uses 'union' and 'combination' to mean $\wedge$, e.g., $[41$, p. 50].)

Proof (i) Consider any decidable property $P$ of natural numbers.

Construct the binary approximation number $x$, as in theorem $6 \mathrm{a}$. 
Define sets $S=\left\{2^{-n} \mid n \in \mathbb{N}\right\}$ and $T=S \cup\{x\}$.

Then $(\{S, T\},=, \subset)$ is a partially ordered set.

Also, $(\{S, T\},=, \subset)$ is a virtually ordered set iff $\neg \neg \exists n P(n) \Rightarrow \exists n P(n)$.

This proves part (i).

(ii) Consider any proposition $A$.

Choose two objects $a, b$ and define binary relations $\equiv, \prec$ on them by

$$
\begin{array}{lll}
a \equiv a, & b \equiv b, & a \neq b, \quad b \neq \equiv a \\
a \nprec a, & b \nprec b, & a \prec b \Leftrightarrow \neg A, \quad b \prec a \Leftrightarrow \neg \neg A .
\end{array}
$$

Then $(\{a, b\}, \equiv, \prec)$ is a virtually ordered set.

Also, $(\{a, b\}, \equiv, \prec)$ is a simply ordered set iff $\neg A \vee \neg \neg A$.

This proves part (ii).

(iii) Consider any proposition $A$.

Choose two objects $a, b$ and define binary relations $\equiv, \prec$ on them by

$$
\begin{array}{lll}
a \equiv a, & b \equiv b, & a \equiv b \Leftrightarrow b \equiv a \Leftrightarrow A \\
a \nprec a, & b \nprec b, & a \prec b \Leftrightarrow \neg A, \quad b \nprec a .
\end{array}
$$

Then $(\{a, b\}, \equiv, \prec)$ is a simply ordered set.

Also, $(\{a, b\}, \equiv, \prec)$ is a virtually ordered set iff $\neg \neg A \Rightarrow A$.

This proves part (iii).

From this may be derived theorem $14 \mathrm{~b}$, the version using the creative subject: for any proposition $A$ construct its direct property $P$ and apply theorem 14a(i), giving a partially ordered set that is virtually ordered only if $A \vee \neg A$ [if axiom 1 is used] or only if $\neg \neg A \vee \neg A$ [if axiom $1^{\prime}$ is used].

Theorem 15a. For any simple order $\prec$ on $\mathbb{R}$ (with $\equiv$ defined as $=)$, and any decidable property $P$ of natural numbers, there exists $x \in \mathbb{R}$ such that

(i) $x \prec-x \wedge x \# 0 \Leftrightarrow \exists n P^{\prime}(2 n)$

(ii) $-x \prec x \wedge x \# 0 \Leftrightarrow \exists n P^{\prime}(2 n+1)$

(iii) $x \# 0 \Leftrightarrow \exists n P(n)$

(iv) $x=0 \Leftrightarrow \neg \exists n P(n)$

(v) $x \neq 0 \Leftrightarrow \neg \neg \exists n P(n)$; and hence

(vi) $\neg \neg \exists n P(n) \Rightarrow \neg \exists n P^{\prime}(2 n) \vee \neg \exists n P^{\prime}(2 n+1)$.

(Brouwer uses part (vi) to show the lack of a simple order on the reduced continuum [41, pp.55-6]. I am modifying his construction inessentially. There is a converse: if the schema $\neg \neg \exists n P(n) \Rightarrow \neg \exists n P^{\prime}(2 n) \vee \neg \exists n P^{\prime}(2 n+1)$ holds then $(\mathbb{R},=, \mathbb{})$ is a simply ordered set.)

Proof Consider any simple order $\prec$ on $\mathbb{R}$ (with $\equiv$ defined as $=$ ), and any decidable property $P$ of natural numbers.

Since $\prec$ is simple we have $\forall n\left(2^{-n} \prec-2^{-n} \vee-2^{-n} \prec 2^{-n}\right)$. 
Define a sequence $a$ of reals by

$$
\forall n \quad a_{n}= \begin{cases}0 & \text { if } \forall i \leq n \neg P(i) \\ 2^{-2 r} & \text { if } 2 r \leq n \text { and } P^{\prime}(2 r) \text { and } 2^{-2 r} \prec-2^{-2 r} \\ -2^{-2 r} & \text { if } 2 r \leq n \text { and } P^{\prime}(2 r) \text { and }-2^{-2 r} \prec 2^{-2 r} \\ -2^{-2 r-1} & \text { if } 2 r+1 \leq n \text { and } P^{\prime}(2 r+1) \text { and } 2^{-2 r-1} \prec-2^{-2 r-1} \\ 2^{-2 r-1} & \text { if } 2 r+1 \leq n \text { and } P^{\prime}(2 r+1) \text { and }-2^{-2 r-1} \prec 2^{-2 r-1}\end{cases}
$$

This is a Cauchy sequence, so define $x=\lim _{n \rightarrow \infty} a_{n}$.

Parts (i-vi) now follow. I shall just give the argument for (vi).

Suppose $\neg \neg \exists n P(n)$.

$$
\begin{aligned}
& \text { By part (v) } x \neq 0, \\
& \text { so }-x \neq x, \\
& \text { so }-x \prec x \vee x \prec-x, \\
& \text { so } \neg(x \prec-x) \vee \neg(-x \prec x), \\
& \text { so by parts (i,ii) } \neg \exists n P^{\prime}(2 n) \vee \neg \exists n P^{\prime}(2 n+1) .
\end{aligned}
$$

This shows that $\neg \neg \exists n P(n) \Rightarrow \neg \exists n P^{\prime}(2 n) \vee \neg \exists n P^{\prime}(2 n+1)$.

This proves the theorem.

The 'b' version of this theorem [17], [41, pp. 54-5] uses the oscillatory property of an arbitrary proposition $A$ to give the conclusion in part (vi) $\neg A \vee \neg \neg A$ [using axioms $\left.2^{\prime}, 3\right]$. This shows the lack of a simple order on the full continuum.

\subsection{Weak Counterexamples to the Basic Properties of the Classical Continuum}

Theorem 16a (weak counterexample to the least upper bound principle). For any decidable property $P$ of natural numbers, there exists a set $S$ of rational numbers such that

(i) $\exists q q \in S$

(ii) $\exists b \in \mathbb{R} \forall q \in S q \leq b$

(iii) if $S$ has a (weak) supremum then $\neg \neg \exists n P(n) \vee \neg \exists n P(n)$

where $l$ is a (weak) supremum of $S$ iff $\forall b \in \mathbb{R}(\forall q \in S q \leq b \Leftrightarrow l \leq b)$.

(This is from $[11$, p. 56].)

Proof Consider any decidable property $P$ of natural numbers.

Define a sequence $a$ of rationals by

$$
a_{0}=1, \quad \forall n a_{n+1}= \begin{cases}\frac{1}{2} a_{n} & \text { if } \neg P^{\prime}(n) \\ 1 & \text { if } P^{\prime}(n)\end{cases}
$$

and define $S=\left\{q \in \mathbb{Q} \mid \exists n q<\sum_{i=0}^{n} a_{i}\right\}$.

(i) and (ii) are easy.

(iii)

Suppose $S$ has a weak supremum, $l$. 
Then $\exists n P(n) \Rightarrow \frac{9}{4} \in S \Rightarrow \frac{9}{4} \leq l$,

and $\neg \exists n P(n) \Rightarrow \forall q \in S q<2 \Rightarrow l \leq 2$.

Now, $2<l \vee l<\frac{9}{4}$,

so $\neg \neg \exists n P(n) \vee \neg \exists n P(n)$.

This shows that if $S$ has a weak supremum then $\neg \neg \exists n P(n) \vee \neg \exists n P(n)$, as required.

This proves the theorem.

The ' $b$ ' form of the theorem uses the conditional property of an arbitrary proposition $A$ to conclude in part (iii) that if $S$ has a weak supremum then $\neg \neg A \vee \neg A$ [using axioms $1^{\prime}, 2^{\prime}$ ]. Brouwer does not consider this.

Theorem 17a (failure of Dedekind cuts always to define a real). For any decidable property $P$ of natural numbers, there exist sets $S, T$ of reals such that

(i) $\exists x x \in S$ and $\exists x x \in T$

(ii) $\forall x \in S \forall y \in T x<y$

(iii) $\neg \exists x \in \mathbb{R}(x \notin S \wedge x \notin T)$

(iv) $S$ has no maximum

(v) if $T$ has a minimum then $\neg \neg \exists n P(n) \vee \neg \exists n P(n)$.

(This is from [11, p. 61].)

Proof Consider any decidable property $P$ of natural numbers.

Define a sequence $a$ of rationals by

$$
\forall n a_{n}= \begin{cases}1-2^{-n-1} & \text { if } \forall i \leq n \neg P(i) \\ 2-2^{-n-1} & \text { if } \exists i \leq n P(i)\end{cases}
$$

and define sets $S=\left\{x \in \mathbb{R} \mid \exists n x<a_{n}\right\}$ and $T=\left\{x \in \mathbb{R} \mid \forall n x \geq a_{n}\right\}$.

(i-iv) are easy.

(v)

Suppose $T$ has a minimum, $M$.

We show $\exists n P(n) \Rightarrow M \geq \frac{3}{2}$,

and $\neg \exists n P(n) \Rightarrow \forall n a_{n}=1-2^{-n-1} \Rightarrow 1 \in T \Rightarrow M \leq 1$.

Now, $1<M \vee M<\frac{3}{2}$,

so $\neg \neg \exists n P(n) \vee \neg \exists n P(n)$.

This shows that if $T$ has a minimum then $\neg \neg \exists n P(n) \vee \neg \exists n P(n)$, as required.

This proves the theorem.

The ' $b$ ' form of the theorem (not considered by Brouwer) uses the conditional property of an arbitrary proposition $A$ to give the conclusion in part (v) that if $T$ has a minimum then $\neg \neg A \vee \neg A$ [using axioms $1^{\prime}, 2^{\prime}$ ]. 
Theorem 18a (weak counterexample to compactness). For any decidable property $P$ of natural numbers, there exists a nested sequence I of closed intervals such that

$$
\exists x x \in \bigcap_{n} I_{n} \Rightarrow \neg \exists n P^{\prime}(2 n+1) \vee \neg \exists n P^{\prime}(2 n) .
$$

(This is from [11, p. 62].)

Proof Consider any decidable property $P$ of natural numbers.

Define a nested sequence $I$ of closed intervals by

$$
\forall n \quad I_{n}= \begin{cases}{\left[-\frac{1}{2}, \frac{1}{2}\right]} & \text { if } \forall i \leq n \neg P(i) \\ {\left[-\frac{1}{2},-\frac{1}{4}\right]} & \text { if } r \leq n \text { and } P^{\prime}(r) \text { and } r \text { is odd } \\ {\left[\frac{1}{4}, \frac{1}{2}\right]} & \text { if } r \leq n \text { and } P^{\prime}(r) \text { and } r \text { is even. }\end{cases}
$$

Suppose $\exists x x \in \bigcap_{n} I_{n}$.

Choose such an $x$.

Then $\exists n P^{\prime}(2 n+1) \Rightarrow x \in\left[-\frac{1}{2},-\frac{1}{4}\right]$, and $\exists n P^{\prime}(2 n) \Rightarrow x \in\left[\frac{1}{4}, \frac{1}{2}\right]$.

Now, $-\frac{1}{4}<x \vee x<\frac{1}{4}$,

so $\neg \exists n P^{\prime}(2 n+1) \vee \neg \exists n P^{\prime}(2 n)$.

This shows that $\exists x x \in \bigcap_{n} I_{n} \Rightarrow \neg \exists n P^{\prime}(2 n+1) \vee \neg \exists n P^{\prime}(2 n)$.

This proves the theorem.

The ' $b$ ' form of the theorem (not considered by Brouwer) uses the oscillatory property of an arbitrary proposition $A$ to give $\exists x x \in \bigcap_{n} I_{n} \Rightarrow \neg \neg A \vee \neg A$ [using axioms $\left.2^{\prime}, 3\right]$.

\subsection{Weak Counterexamples in the Theory of Real Functions}

Def ${ }^{\text {n }}$. A real number $a$ is a point of positive discontinuity of a function $f: \mathbb{R} \rightarrow \mathbb{R}$ iff there exists a sequence $u$ of real numbers such that $u_{n} \rightarrow a$ as $n \rightarrow \infty$ and $\exists \epsilon \in \mathbb{Q}^{+} \forall n\left|f\left(u_{n}\right)-f(a)\right| \geq \epsilon$.

$f$ is negatively continuous iff it does not have a point of positive discontinuity.

(Negative continuity is equivalent to sequential nondiscontinuity [2].)

$f$ is continuous iff $\forall a \forall \epsilon \in \mathbb{Q}^{+} \exists \delta \in \mathbb{Q}^{+} \forall x(|x-a|<\delta \Rightarrow|f(x)-f(a)|<\epsilon)$.

Any function $f$ is required to be extensional, which means that it satisfies $\forall x, y(x=y \Rightarrow f(x)=f(y))$. A function $f$ is strongly extensional iff it satisfies $\forall x, y(f(x) \# f(y) \Rightarrow x \# y)$.

Theorem 19a (negative continuity theorem). For any $f: \mathbb{R} \rightarrow \mathbb{R}$ having a point of positive discontinuity, and any decidable property $P$ of natural numbers,

(i) $\neg \exists n P(n) \vee \neg \neg \exists n P(n)$

(ii) if $f$ is strongly extensional then $\exists n P(n) \vee \neg \exists n P(n)$. 
(Brouwer uses part (i) to show that all functions are negatively continuous [41, pp. 80-1]. The converses hold: if one assumes $\neg \exists n P(n) \vee \neg \neg \exists n P(n)$ then one can define a function with one removable discontinuity, and if one assumes $\exists n P(n) \vee \neg \exists n P(n)$ one can define a step function that is strongly extensional. Also, Ishihara [28] shows that, assuming a weak form of Markov's principle, every function $\mathbb{R} \rightarrow \mathbb{R}$ is strongly extensional, and every negatively continuous function $\mathbb{R} \rightarrow \mathbb{R}$ is sequentially continuous, i.e., $u_{n} \rightarrow a$ as $n \rightarrow \infty$ implies $f\left(u_{n}\right) \rightarrow f(a)$ as $n \rightarrow \infty$. Therefore if one assumes the weak Markov's principle together with $\neg \forall P(\exists n P(n) \vee \neg \exists n P(n))$ then one infers that all functions $\mathbb{R} \rightarrow \mathbb{R}$ are sequentially continuous.)

Proof Consider any function $f: \mathbb{R} \rightarrow \mathbb{R}$ having a point of positive discontinuity $a$.

Choose a real sequence $u$ such that $u_{n} \rightarrow a$ as $n \rightarrow \infty$ and a rational $\epsilon>0$ such that $\forall n\left|f\left(u_{n}\right)-f(a)\right| \geq \epsilon$.

Consider any decidable property $P$ of natural numbers.

Construct the checking-number $x \in \mathbb{R}$ of the drift $(a, u)$ through $P$.

Since $0<\epsilon$ we have $0<|f(x)-f(a)| \vee|f(x)-f(a)|<\epsilon$. (1)

Now, $\exists n P(n) \Rightarrow \exists n x=u_{n} \Rightarrow|f(x)-f(a)| \geq \epsilon$,

so $|f(x)-f(a)|<\epsilon \Rightarrow \neg \exists n P(n)$. (2)

(i)

Now, $\neg \exists n P(n) \Rightarrow x=a \Rightarrow|f(x)-f(a)|=0$,

so $0<|f(x)-f(a)| \Rightarrow \neg \neg \exists n P(n)$. (3)

By (1), (2) and (3), $\neg \exists n P(n) \vee \neg \neg \exists n P(n)$.

(ii)

Suppose $f$ is strongly extensional.

Then $0<|f(x)-f(a)| \Rightarrow f(x) \# f(a) \Rightarrow x \# a \Rightarrow \exists n P(n)$. (4)

By (1), (2) and (4), $\exists n P(n) \vee \neg \exists n P(n)$.

This shows that if $f$ is strongly extensional then $\exists n P(n) \vee \neg \exists n P(n)$.

This proves (i) and (ii) for any decidable property $P$ of natural numbers.

This proves the theorem.

The ' $b$ ' form of the theorem uses the conditional property of an arbitrary proposition $A$ to give $\neg A \vee \neg \neg A$ [using axioms $1^{\prime}, 2^{\prime}$ ], and $A \vee \neg A$ if $f$ is strongly extensional [using axioms $1,2^{\prime}$ ]. Brouwer does not consider this but instead gives a completely different proof of the negative continuity theorem in [8]; see theorem 30 below.

Theorem 20a (weak counterexample to the intermediate value theorem). For any decidable property $P$ of natural numbers, there exists a continuous function $f$ : $[-2,2] \rightarrow \mathbb{R}$ such that $f(-2)>0$ and $f(2)<0$ but

$$
\exists c \in(-2,2) f(c)=0 \Rightarrow \neg \exists n P^{\prime}(2 n) \vee \neg \exists n P^{\prime}(2 n+1) .
$$


(This is from [12, p. 445]. Conversely, as Bridges \& Richman point out [2, ch. 3, theorem 2.4], given the schema $\neg \exists n P^{\prime}(2 n) \vee \neg \exists n P^{\prime}(2 n+1)$ the intermediate value theorem is easily proved.)

Proof Consider any decidable property $P$ of natural numbers.

Construct the oscillatory binary shrinking number $b \in \mathbb{R}$ as in theorem $7 \mathrm{a}$, and define $a=|b|$ (in fact, $a$ is just the binary approximation number constructed in theorem 6a).

Construct a continuous function $f:[-2,2] \rightarrow \mathbb{R}$ such that

$$
f(-2)=1, \quad f(-1)=a, \quad f(1)=b, \quad f(2)=-1,
$$

with $f$ linear on $[-2,-1]$ and on $[-1,1]$ and on $[1,2]$.

Suppose $\exists c \in(-2,2) f(c)=0$.

Choose such a $c$.

Then $\exists n P^{\prime}(2 n) \Rightarrow b=a>0 \Rightarrow c>1$,

and $\exists n P^{\prime}(2 n+1) \Rightarrow b=-a<0 \Rightarrow c=0$.

But $1>c \vee c>0$,

so $\neg \exists n P^{\prime}(2 n) \vee \neg \exists n P^{\prime}(2 n+1)$.

This shows that $\exists c \in(-2,2) f(c)=0 \Rightarrow \neg \exists n P^{\prime}(2 n) \vee \neg \exists n P^{\prime}(2 n+1)$.

This proves the theorem.

The ' $b$ ' form of the theorem (not considered by Brouwer) uses the oscillatory property of an arbitrary proposition $A$ to give $\exists c \in(-2,2) f(c)=0 \Rightarrow \neg A \vee \neg \neg A$ [using axioms $2^{\prime}, 3$ ].

Theorem 21a (weak counterexample to existence of local maximum of continuous functions). For any decidable property $P$ of natural numbers, there exists a continuous function $f:[0,1] \rightarrow \mathbb{R}$ such that if $f$ has a local maximum then $\neg \exists n P^{\prime}(2 n) \vee \neg \exists n P^{\prime}(2 n+1)$.

(This is an early example from 1923 [5]. Brouwer used a particular fleeing property involving the occurrence of 0123456789 in the digits of $\pi$, which I have generalised to an arbitrary fleeing property. Brouwer simply asserted that the function 'possesses no maximum', whereas I derive a specific constructively invalid logical schema from the assumption of a local maximum; to do this I have had to simplify his argument, replacing his use of an enumeration of all binary fractions in $(0,1)$ simply by $\frac{1}{4}$ and $\frac{3}{4}$.)

Proof Consider any decidable property $P$ of natural numbers.

For each $n$, define $h_{2 n}:[0,1] \rightarrow \mathbb{R}$ such that $h_{2 n}(0)=0, h_{2 n}\left(\frac{1}{4}\right)=2^{-n}$, $h_{2 n}(1)=0$, and $h_{2 n}$ is linear between these points;

and define $h_{2 n+1}:[0,1] \rightarrow \mathbb{R}$ such that $h_{2 n+1}(0)=0, h_{2 n+1}\left(\frac{3}{4}\right)=2^{-n}$, $h_{2 n+1}(1)=0$, and $h_{2 n+1}$ is linear between these points.

For each $n$, define $g_{n}$ as $h_{n}$ if $P^{\prime}(n)$, and as the zero function otherwise.

Define $f:[0,1] \rightarrow \mathbb{R}$ as $\sum_{n=0}^{\infty} g_{n}$. 
Then $\forall x, y \in[0,1]|f(x)-f(y)| \leq 4|x-y|$, so $f$ is continuous.

Suppose $f$ has a local maximum, attained at $x \in[0,1]$.

If $\exists n P^{\prime}(2 n)$ then $f=h_{2 n}$, so $x=\frac{1}{4}$.

If $\exists n P^{\prime}(2 n+1)$ then $f=h_{2 n+1}$, so $x=\frac{3}{4}$.

But $\frac{1}{4}<x \vee x<\frac{3}{4}$,

so $\neg \exists n P^{\prime}(2 n) \vee \neg \exists n P^{\prime}(2 n+1)$.

This shows that if $f$ has a local maximum then $\neg \exists n P^{\prime}(2 n) \vee \neg \exists n P^{\prime}(2 n+1)$.

This proves the theorem.

The 'b' form of the theorem (not considered by Brouwer) uses the oscillatory property of an arbitrary proposition $A$ to show that if $f$ has a local maximum then $\neg A \vee \neg \neg A$ [using axioms $2^{\prime}, 3$ ].

An alternative approach is to show that the proposition that every continuous function on $[0,1]$ has a local maximum implies $\forall x, y(x \leq y \vee y \leq x)$, which is refuted by theorem $7 \mathrm{a}$.

Def $^{\mathbf{n}}$. (Weak and strong differentiation [23])

$c$ is a weak derivative of $f$ at $a, f_{\text {weak }}^{\prime}(a)=c$, iff

$\neg \exists n \forall m \exists p, q\left(p<a<q \wedge q-p<2^{-m} \wedge\left|\frac{f(q)-f(p)}{q-p}-c\right|>2^{-n}\right) ;$

$c$ is a strong derivative of $f$ at $a, f_{\text {strong }}^{\prime}(a)=c$, iff

$$
\forall n \exists m \forall p, q\left(p<a<q \wedge q-p<2^{-m} \Rightarrow\left|\frac{f(q)-f(p)}{q-p}-c\right|<2^{-n}\right) .
$$

Note that a strong derivative is unique, and that $f_{\text {strong }}^{\prime}(a)=c$ implies $f_{\text {weak }}^{\prime}(a)=$ $c$; but it does not seem possible to prove that a weak derivative is unique.

$f$ is weakly differentiable at $a$ iff it has a weak derivative at $a$.

$f$ is strongly differentiable at $a$ iff it has a strong derivative at $a$.

These concepts are defined for functions $f: \mathbb{R} \rightarrow \mathbb{R}$ but can be adapted to functions on an interval in the usual way.

In future if I omit the qualification 'weak' or 'strong' then the argument works for both kinds of differentiation.

Theorem 22a. For any decidable property $P$ of natural numbers, there exists a strictly increasing, uniformly continuous function $f:[0,1] \rightarrow \mathbb{R}$ such that if $f$ is differentiable at any point then $\neg \exists n P(n) \vee \neg \neg \exists n P(n)$.

(This is from [22]. It supersedes an earlier weak counterexample in [5], which was faulty.)

Proof Consider any decidable property $P$ of natural numbers. 
For each $n>0$, define a 'sawtooth' function $\chi_{n}:[0,1] \rightarrow \mathbb{R}$ such that

$$
\begin{aligned}
\forall i \in\{0, \ldots n\} \quad \chi_{n}\left(\frac{i}{n}\right) & =0 \\
\forall i \in\{0, \ldots n-1\} \quad \chi_{n}\left(\frac{2 i+1}{2 n}\right) & =\frac{1}{4 n}
\end{aligned}
$$

with $\chi_{n}$ linear between these points.

For each $n>0$, define a function $\psi_{n}:[0,1] \rightarrow \mathbb{R}$ such that

$$
\forall x \in[0,1] \quad \psi_{n}(x)= \begin{cases}\chi_{n}(x) & \text { if } P^{\prime}(n-1) \\ 0 & \text { otherwise. }\end{cases}
$$

Define $f:[0,1] \rightarrow \mathbb{R}$ by $\forall x \in[0,1] f(x)=x+\sum_{n=1}^{\infty} \psi_{n}(x)$.

Then $f$ is strictly increasing.

Also, $\forall x, y \in[0,1]|f(x)-f(y)| \leq \frac{3}{2}|x-y|$,

so $f$ is uniformly continuous.

Suppose there exists a point $a \in[0,1]$ at which $f$ is differentiable.

Then $\neg \exists n P(n) \Rightarrow \forall x \in[0,1] f(x)=x \Rightarrow f^{\prime}(a)=1$; and we can show $f^{\prime}(a) \# \frac{1}{2} \wedge f^{\prime}(a) \# \frac{3}{2} \Rightarrow \neg \exists n P(n)$.

Now, $f^{\prime}(a) \# 1 \vee f^{\prime}(a) \# \frac{1}{2}$ and $f^{\prime}(a) \# 1 \vee f^{\prime}(a) \# \frac{3}{2}$, so $\neg \exists n P(n) \vee \neg \neg \exists n P(n)$.

This shows that if $f$ is differentiable at any point then $\neg \exists n P(n) \vee \neg \neg \exists n P(n)$. This proves the theorem.

Brouwer [24] also considers the ' $b$ ' form of the theorem. For any proposition $A$ the conditional property is used to show that if $f$ is differentiable at any point then $\neg A \vee \neg \neg A$ [using axioms $1^{\prime}, 2^{\prime}$ ].

Theorem 23a. For any decidable property $P$ of natural numbers, there exists a function $f: \mathbb{R} \rightarrow \mathbb{R}$ such that

(i) $f_{\text {strong }}^{\prime}(0)=0 \Leftrightarrow \exists n P(n)$

(ii) $\quad\left(f_{\text {weak }}^{\prime}(0)=0 \Rightarrow f_{\text {strong }}^{\prime}(0)=0\right) \Rightarrow(\neg \neg \exists n P(n) \Rightarrow \exists n P(n))$.

(This shows the difference between weak and strong derivatives.)

Proof For each $n$, define $\omega_{n}: \mathbb{R} \rightarrow \mathbb{R}$ by

$$
\begin{array}{cl}
\forall x \leq-2^{-n+1} & \omega_{n}(x)=0 \\
\forall x \in\left[-2^{-n+1},-2^{-n}\right] & \omega_{n}(x)=\sqrt{\left(x+2^{-n+1}\right)\left(-2^{-n}-x\right)} \\
\forall x \in\left[-2^{-n}, 2^{-n}\right] & \omega_{n}(x)=0 \\
\forall x \in\left[2^{-n}, 2^{-n+1}\right] & \omega_{n}(x)=\sqrt{\left(x-2^{-n}\right)\left(2^{-n+1}-x\right)} \\
\forall x \geq 2^{-n+1} & \omega_{n}(x)=0 .
\end{array}
$$

Consider any decidable property $P$ of natural numbers. 
For any $n$, define $\zeta_{n}: \mathbb{R} \rightarrow \mathbb{R}$ by

$$
\forall x \in \mathbb{R} \zeta_{n}(x)= \begin{cases}\omega_{n}(x) & \text { if } \forall i \leq n \neg P(i) \\ 0 & \text { if } \exists i \leq n P(i) .\end{cases}
$$

Define $f: \mathbb{R} \rightarrow \mathbb{R}$ by $\forall x \in \mathbb{R} f(x)=\sum_{n=1}^{\infty} \zeta_{n}(x)$.

(i)

Suppose $f_{\text {strong }}^{\prime}(0)=0$.

Choose $m$ such that $\forall p, q\left(p<0<q \wedge q-p<2^{-m} \Rightarrow\left|\frac{f(q)-f(p)}{q-p}\right|<2^{-3}\right)$.

Choose $p=-2^{-m-2}$ and $q=\frac{3}{2} 2^{-m-2}$.

Suppose $\forall i \leq m+2 \neg P(i)$.

Then $f(p)=0$ and $f(q)=\omega_{m+2}\left(\frac{3}{2} 2^{-m-2}\right)=\frac{1}{2} 2^{-m-2}$, so

$$
\left|\frac{f(q)-f(p)}{q-p}\right|=\left|\frac{\frac{1}{2} 2^{-m-2}-0}{\frac{3}{2} 2^{-m-2}+2^{-m-2}}\right|=\frac{1}{5} \nless 2^{-3},
$$

which is a contradiction.

This shows that $\exists i \leq m+2 P(i)$.

This shows that $f_{\text {strong }}^{\prime}(0)=0 \Rightarrow \exists n P(n)$.

Conversely, $\exists n P(n) \Rightarrow f_{\text {strong }}^{\prime}(0)=0$.

Hence $f_{\text {strong }}^{\prime}(0)=0 \Leftrightarrow \exists n P(n)$, as required.

(ii)

By part (i), $\exists n P(n) \Rightarrow f_{\text {strong }}^{\prime}(0)=0 \Rightarrow f_{\text {weak }}^{\prime}(0)=0$,

so, contraposing twice, $\neg \neg \exists n P(n) \Rightarrow f_{\text {weak }}^{\prime}(0)=0$.

Part (i) also says, $f_{\text {strong }}^{\prime}(0)=0 \Rightarrow \exists n P(n)$.

So $\left(f_{\text {weak }}^{\prime}(0)=0 \Rightarrow f_{\text {strong }}^{\prime}(0)=0\right) \Rightarrow(\neg \neg \exists n P(n) \Rightarrow \exists n P(n))$, as required.

This proves the theorem.

In this case Brouwer considers only the ' $b$ ' form of the theorem [23]. For any proposition $A$ the direct property is used to show that if $f_{\text {weak }}^{\prime}(0)=0 \Rightarrow$ $f_{\text {strong }}^{\prime}(0)=0$ then $A \vee \neg A$ [using axioms 1,2'] or $\neg \neg A \vee \neg A$ [using axioms $\left.1^{\prime}, 2^{\prime}\right]$; Brouwer seems to be doing the former, since he states his argument in terms of an unjudgeable proposition rather than an untestable one.

Theorem 24a (weak counterexample to Rolle's theorem). For any decidable property $P$ of natural numbers, there exists a continuously differentiable function $f:[-3,3] \rightarrow \mathbb{R}$ such that $f(-3)=f(3)$ and

$$
\exists c \in(-3,3) f^{\prime}(c)=0 \Rightarrow \neg \exists n P^{\prime}(2 n+1) \vee \neg \exists n P^{\prime}(2 n) .
$$

(This is from [12, p. 445].)

Proof Consider any decidable property $P$ of natural numbers. 
Construct the oscillatory binary shrinking number $b \in \mathbb{R}$ as in theorem $7 \mathrm{a}$, and define $a=|b|$.

Construct a continuous function $g:[-3,3] \rightarrow \mathbb{R}$ such that

$$
\begin{aligned}
& g(-3)=1, \quad g(-2)=a, \quad g(-1)=b, \quad g(0)=-1, \\
& g(1)=-a, \quad g(2)=-b, \quad g(3)=1 \text {, }
\end{aligned}
$$

with $g$ linear on $[-3,-2]$ and $[-2,-1]$ and $[-1,0]$ and $[0,1]$ and $[1,2]$ and $[2,3]$, and construct a piecewise quadratic function $f:[-3,3] \rightarrow \mathbb{R}$ such that $f(3)=$ $0=f(-3)$ and $f^{\prime}=g$.

Suppose $\exists c \in(-3,3) f^{\prime}(c)=0$.

Choose such a $c$.

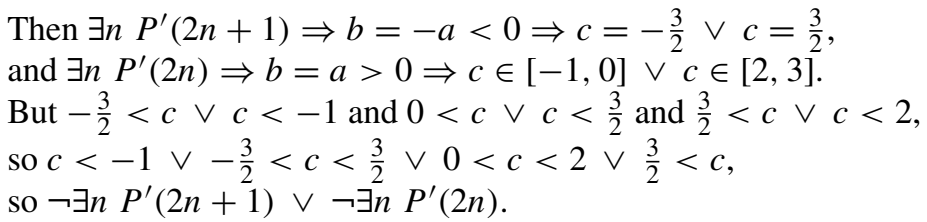

This shows that $\exists c \in(-3,3) f^{\prime}(c)=0 \Rightarrow \neg \exists n P^{\prime}(2 n+1) \vee \neg \exists n P^{\prime}(2 n)$.

This proves the theorem.

The ' $b$ ' form of the theorem (not considered by Brouwer) uses the oscillatory property of an arbitrary proposition $A$ to give $\exists c \in(-3,3) f^{\prime}(c)=0 \Rightarrow \neg \neg A \vee$ $\neg A$ [using axioms $2^{\prime}, 3$ ].

\subsection{Weak Counterexamples in Topology}

Theorem 25a (weak counterexample to the Heine-Borel theorem). For any decidable property $P$ of natural numbers, there exists a closed and bounded set $S \subseteq \mathbb{R}$ such that

(i) $S \subseteq \bigcup_{n \in \mathbb{N}} U_{n}$, for a countably indexed family ${ }_{n}\left(U_{n}\right)$ of open sets,

(ii) if there exists a finite set $\left\{n_{1}, \ldots n_{k}\right\} \subseteq \mathbb{N}$ such that $S \subseteq \bigcup_{i=1}^{k} U_{n_{i}}$ then $\exists n P(n) \vee \neg \exists n P(n)$.

(Brouwer $[5,21]$ stated this originally for a particular fleeing property involving the occurrence of 0123456789 in the digits of $\pi$, which I have generalised to an arbitrary fleeing property. There are subtleties in the interpretation of the word 'closed'. I have taken the simplest proof from [21]; a set $S$ is closed iff $x_{n} \rightarrow l$ as $n \rightarrow \infty$ and $\forall n x_{n} \in S$ imply $l \in S$. The counterexample works by constructing a set $S$ that is not 'located'; see [2, 21] for more on this.)

Proof Consider any decidable property $P$ of natural numbers.

Define a set $S=\left\{u_{n} \mid n \in \mathbb{N}\right\}$, where $\forall n u_{n}=\left\{\begin{array}{ll}-1 & \text { if } \exists i \leq n P(i) \\ 1 & \text { if } \forall i \leq n \neg P(i)\end{array}\right.$. 
Then $S$ is closed and bounded.

(i)

For each $n$ choose $U_{n}=\left(u_{n}-1, u_{n}+1\right)$.

Then $S \subseteq \bigcup_{n \in \mathbb{N}} U_{n}$.

(ii)

Suppose $S \subseteq \bigcup_{i=1}^{k} U_{n_{i}}$, for some finite subset $\left\{n_{1}, \ldots n_{k}\right\} \subseteq \mathbb{N}$.

Choose $N=\max \left\{n_{1}, \ldots n_{k}\right\}$.

Suppose $\neg \exists n \leq N P(n)$.

Then $u_{n_{1}}, \ldots u_{n_{k}}$ all equal 1 ,

so $\bigcup_{i=1}^{k} U_{n_{i}}=(0,2)$,

so $-1 \notin S$, so $\neg \exists n P(n)$.

This shows that $\neg \exists n \leq N P(n) \Rightarrow \neg \exists n P(n)$.

Since we have $\exists n \leq N P(n) \vee \neg \exists n \leq N P(n)$ then $\exists n P(n) \vee \neg \exists n P(n)$.

This shows that if $S \subseteq \bigcup_{i=1}^{k} U_{n_{i}}$, for some $\left\{n_{1}, \ldots n_{k}\right\} \subseteq \mathbb{N}$, then $\exists n P(n) \vee$ $\neg \exists n P(n)$.

This proves the theorem.

The ' $b$ ' form of the theorem (not considered by Brouwer) uses the conditional property of an arbitrary proposition $A$ to show that if ${ }_{n}\left(U_{n}\right)$ has a finite subcover then $A \vee \neg A$ [using axioms $1,2^{\prime}$ ].

A version of the Heine-Borel theorem can however be proved if the fan theorem is assumed [38, vol. 1, p. 305]

Def $^{\mathbf{n}}$. A real number $x$ is a point of accumulation of a real set $S, \operatorname{acc}_{S}(x)$, iff

$$
\forall a, b \in \mathbb{R}\left(a<x<b \Rightarrow \exists^{\infty} p \in(a, b) p \in S\right) .
$$

Def $^{\text {n }}$. A real set $S$ is bounded in number, $b n(S)$, iff there exists $n$ such that $S$ has no subset of cardinality $n$.

The A form of the Bolzano-Weierstrass theorem says that every infinite $S \subseteq[0,1]$ has a point of accumulation.

The B form of the Bolzano-Weierstrass theorem says that every $S \subseteq[0,1]$ without a point of accumulation is bounded in number.

Theorem 26a (weak counterexample to the $\mathbf{A}$ form). For any decidable property $P$ of natural numbers, there exists an infinite set $S \subseteq[0,1]$ such that

$$
\exists x \operatorname{acc}_{S}(x) \Rightarrow \exists n P(n) \vee \neg \exists n P(n) .
$$

(This is from [18, p. 517].)

Proof Consider any decidable property $P$ of natural numbers.

Define an infinite set $S=\left\{a_{n} \mid n>0\right\} \subseteq[0,1]$, where

$$
\forall n>0 a_{n}= \begin{cases}\frac{1}{3}+2^{-n} & \text { if } \forall i \leq n \neg P(i) \\ \frac{1}{4}+2^{-n} & \text { if } \exists i \leq n P(i) .\end{cases}
$$


We show by cases that $\forall x \in \mathbb{R}\left(x \# \frac{1}{3} \wedge x \# \frac{1}{4} \Rightarrow \neg \operatorname{acc}_{S}(x)\right)$.

Hence $\forall x \in \mathbb{R}\left(\operatorname{acc}_{S}(x) \Rightarrow x=\frac{1}{4} \vee x=\frac{1}{3}\right)$.

Suppose $S$ has a point of accumulation, $x$.

Then $x=\frac{1}{4} \vee x=\frac{1}{3}$.

We show that $x=\frac{1}{4} \Rightarrow \exists n P(n)$, and $x=\frac{1}{3} \Rightarrow \neg \exists n P(n)$.

Thus $\exists n P(n) \vee \neg \exists n P(n)$.

This shows that $\exists x \operatorname{accs}_{S}(x) \Rightarrow \exists n P(n) \vee \neg \exists n P(n)$.

This proves the theorem.

The ' $b$ ' form of the theorem (not considered by Brouwer) uses the conditional property of an arbitrary proposition $A$ to give $\exists x \operatorname{accs}_{S}(x) \Rightarrow \neg \neg A \vee \neg A$ [using axioms $\left.1^{\prime}, 2^{\prime}\right]$.

Theorem 27a (weak counterexample to the $\mathbf{B}$ form). For any decidable property $P$ of natural numbers, there is a set $S \subseteq[0,1]$ such that

(i) $b n(S) \Rightarrow \exists n P(n)$

(ii) $\exists n P(n) \Rightarrow \neg \exists x \operatorname{acc}_{S}(x)$

(iii) $\left(\neg \exists x \operatorname{acc}_{S}(x) \Rightarrow b n(S)\right) \Rightarrow(\neg \neg \exists n P(n) \Rightarrow \exists n P(n))$.

Proof Consider any decidable property $P$ of natural numbers.

Define $S=\left\{2^{-n} \mid \forall i \leq n \neg P(i)\right\} \subseteq[0,1]$. (This is a simplification of Brouwer's construction.)

(i)

Suppose $S$ is bounded in number.

Choose $k$ such that $S$ has no subset of cardinality $k$.

If $\forall i \leq k-1 \neg P(i)$ then $\left\{2^{0}, 2^{-1}, \ldots 2^{-k+1}\right\}$ is a subset of $S$ of cardinality $k$, so $\exists i \leq k-1 P(i)$.

This shows that $b n(S) \Rightarrow \exists n P(n)$, as required.

(ii)

Suppose $\exists n P(n)$.

Choose the least $r$ such that $P(r)$.

Then $S=\left\{2^{0}, 2^{1}, \ldots 2^{-r+1}\right\}$,

so $\neg \exists x \operatorname{accs}_{S}(x)$.

This shows that $\exists n P(n) \Rightarrow \neg \exists x \operatorname{acc}_{S}(x)$, as required.

(iii) follows from (i) and the double contrapositive of (ii).

This proves the theorem.

This time it is the ' $b$ ' form of the theorem that Brouwer considers [18, p. 517]. For any proposition $A$ the direct property is used to construct $S \subseteq[0,1]$ that has no point of accumulation [using axiom $2^{\prime}$ ], and if $b n(S)$ then $A \vee \neg A$ [using axiom 1] 
or $\neg \neg A \vee \neg A$ [using axiom $1^{\prime}$ ]; Brouwer seems to be doing the latter, since he considers an untestable $A$.

Theorem 28a (weak counterexample to Brouwer's fixed-point theorem). Define $Q=[-1,1] \times[-1,1]$, a square in $\mathbb{R}^{2}$. For any decidable property $P$ of natural numbers, there is a uniformly continuous injective function $f: Q \rightarrow Q$ such that

(i) $\neg \neg \exists n P(n) \Rightarrow \exists^{\leq 1} p \in Q f(p)=p$,

(ii) $\exists p \in Q f(p)=p \Rightarrow \neg \exists r P^{\prime}(2 r) \vee \neg \exists r P^{\prime}(2 r+1)$.

(This is a weak counterexample to:

every uniformly continuous injective function from $Q$ into $Q$ has a fixed point; every uniformly continuous injective function from $Q$ into $Q$ with at most one fixed point has a fixed point.)

Proof Consider any decidable property $P$ of natural numbers.

Choose points $a=\left(-\frac{1}{2}, 0\right)$ and $b=\left(\frac{1}{2}, 0\right)$ in $Q$.

For any $n$, define a function $t_{a n}: Q \rightarrow Q$ as follows. Represent each point of $Q$ by polar coordinates $(r, \theta)$ based at $a$. The point with polar representation $(r, \theta)$ maps to the point with polar representation $\left(k_{\theta} r, \theta+\tan ^{-1} 2^{-n}\right)$, where $k_{\theta}$ is chosen so that any point on the boundary of $Q$ maps to another point on the boundary.

This defines $t_{a n}$ on each point of $Q$ apart from $a$. It is extended to the whole of $Q$ by continuity.

Then, for each $n, t_{a n}$ is a bijection from $Q$ to itself, with $a$ as its only fixed point, satisfying $\forall p, q \in Q\left\|t_{a n}(p)-t_{a n}(q)\right\| \leq 51\|p-q\|$, and so is uniformly continuous.

Moreover, $\forall m, n \forall p \in Q\left\|t_{a m}(p)-t_{a n}(p)\right\| \leq \frac{13}{4}\left|2^{-m}-2^{-n}\right|$ and $\forall n \forall p \in Q\left\|p-t_{\text {an }}(p)\right\| \leq \frac{13}{4} 2^{-n}$.

For any $n$, define a function $t_{b n}: Q \rightarrow Q$ similarly, but using a polar representation based at $b$. It has similar properties to $t_{a n}$.

Define a sequence $t$ of functions from $Q$ to $Q$ by

$$
\forall n t_{n}= \begin{cases}i d & \text { if } \forall i \leq n \neg P(i) \\ t_{a r} & \text { if } 2 r \leq n \text { and } P^{\prime}(2 r) \\ t_{b r} & \text { if } 2 r+1 \leq n \text { and } P^{\prime}(2 r+1) .\end{cases}
$$

For each $n, t_{n}$ is bijective and uniformly continuous.

Now, $\forall m, n\left(m<n \Rightarrow \forall p \in Q\left\|t_{m}(p)-t_{n}(p)\right\| \leq \frac{13}{4} 2^{-\lceil m / 2\rceil}\right)$,

so, for each $p \in \mathbb{Q}$, the sequence ${ }_{n}\left(t_{n}(p)\right)$ is Cauchy, and hence convergent.

Define $f: \mathbb{Q} \rightarrow \mathbb{Q}$ by $\forall p \in \mathbb{Q} f(p)=\lim _{n \rightarrow \infty} t_{n}(p)$.

Then $\forall m \forall p \in Q\left\|t_{m}(p)-f(p)\right\| \leq \frac{13}{4} 2^{-\lceil m / 2\rceil}$,

so $t_{m} \rightarrow f$ uniformly as $m \rightarrow \infty$,

so $f$ is uniformly continuous.

$f$ is also injective.

(i)

Suppose $\neg \neg \exists n P(n)$. 
Consider any fixed points $p, q$ of $f$.

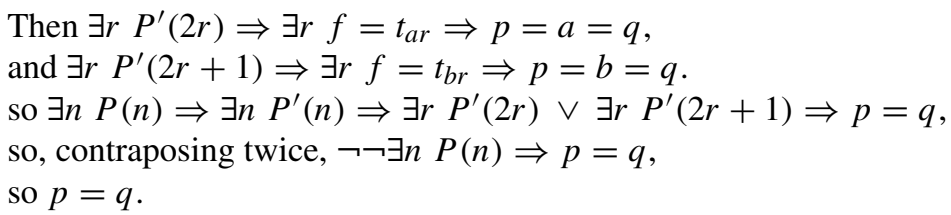

This shows that $\exists \leq 1 p \in Q f(p)=p$.

This shows that $\neg \neg \exists n P(n) \Rightarrow \exists^{\leq 1} p \in Q f(p)=p$, as required.

(ii)

Suppose $\exists p \in Q f(p)=p$.

Choose such a fixed point, $p$.

Then $\exists r P^{\prime}(2 r) \Rightarrow \exists r f=t_{a r} \Rightarrow p=a$;

equivalently, $p \# a \Rightarrow \neg \exists r P^{\prime}(2 r)$.

Similarly, $p \# b \Rightarrow \neg \exists r P^{\prime}(2 r+1)$.

Hence $p \# a \vee p \# b \Rightarrow \neg \exists r P^{\prime}(2 r) \vee \neg \exists r P^{\prime}(2 r+1)$.

Since $\forall p(p \# a \vee p \# b)$, this gives $\neg \exists r P^{\prime}(2 r) \vee \neg \exists r P^{\prime}(2 r+1)$.

This shows that $\exists p \in Q f(p)=p \Rightarrow \neg \exists r P^{\prime}(2 r) \vee \neg \exists r P^{\prime}(2 r+1)$, as required.

This proves the theorem.

Brouwer considers only the ' $b$ ' form of this theorem [19]: for any proposition $A$ the oscillatory property is used to give the conclusions

(i) $\quad \exists^{\leq 1} p \in Q f(p)=p, \quad$ [using axiom $2^{\prime}$ ]

(ii) $\exists p \in Q f(p)=p \Rightarrow \neg A \vee \neg \neg A \quad$ [using axioms $2^{\prime}, 3$ ].

Note that $\exists \leq 1 p \in Q f(p)=p$ is understood as $\forall p, q \in Q(f(p)=p \wedge$ $f(q)=q \Rightarrow p=q)$. A slight alteration gives the opposite result: Veldman [45] shows that, assuming the fan theorem, every uniformly continuous function $f: Q \rightarrow$ $Q$ such that $\forall p, q \in Q(p \# q \Rightarrow f(p) \# p \vee f(q) \# q)$ has a fixed point.

\section{Are Weak Counterexamples Genuine Proofs or Just Plausibility Arguments?}

Brouwer presents his weak counterexamples as dependent on the existence of a fleeing property or 'a mathematical assertion so far neither judged nor recognised as judgeable' or 'a mathematical assertion so far neither tested nor recognised as testable'. This gives them a provisional or subjective appearance.

Does Brouwer think of them as proper mathematical proofs? Occasionally he refers to them as 'theorems' [41, p. 80]. In [12, p. 445] he speaks of 'refuting' classical theorems by weak counterexamples. In [14, p.491] he says, 'To give some examples refuting the principle of the excluded third and its corollaries ...'. In [18] he speaks of 'refutation' of the two forms of the Bolzano-Weierstrass theorem. These expressions suggest that he regards them as definite mathematical results. 
Sometimes, however, he uses weaker language. In [11] he describes the finding of a simple ordering of the continuum as hoffnungslos (hopeless), on account of the weak counterexample. In [14, pp. 493-4] he twice says, 'there seems to be little hope for reducing [relations such as inequality] to a constructive property'. The reason for the vagueness here is not that weak counterexamples are intrinsically vague but that he is trying to show that $x \neq y$ cannot be defined by a positive condition; he has shown that $x \neq y$ is not equivalent to $x \# y$, but he has not considered other possible positive conditions it might be equivalent to.

In my view the weak counterexamples are rigorous proofs of precise mathematical results (given the creative subject axioms assumed). They gain in precision when one states them for all properties, not just fleeing ones. For example, theorem 6a shows that

$$
\forall P \exists x \in \mathbb{R}(x \# 0 \vee x=0 \Rightarrow \exists n P(n) \vee \neg \exists n P(n))
$$

and consequently

$$
\forall x \in \mathbb{R}(x \# 0 \vee x=0) \Rightarrow \forall P(\exists n P(n) \vee \neg \exists n P(n)) .
$$

Thus, if we had a proof of $\forall x \in \mathbb{R}(x \# 0 \vee x=0)$, we could thereby obtain a proof of the principle of excluded middle for $\Sigma_{1}$ propositions.

This sort of result is analogous to proving a problem $N P$-complete. It shows that $\forall x \in \mathbb{R}(x \# 0 \vee x=0)$ is a member of a certain class of propositions of recognised intractability, whose solution would revolutionise mathematical knowledge. This is a definite and valuable result, not to be considered a mere heuristic argument. It can be understood even by a classical mathematician.

Just the same remarks apply to the versions of the weak counterexamples with the creative subject.

(This assumes, of course, that one has no predicativist objection to quantifying over all properties of natural numbers or all propositions.)

If a general method of solving all mathematical problems is ever invented then all my theorems in $\$ 5$ remain valid but they lose all value, just as if a proof of $P=N P$ is ever found then all proofs of $N P$-hardness remain valid but lose all value.

\section{What are the Right Axioms for the Creative Subject?}

I am not concerned here with the soundness of the axioms but with their usefulness in deriving the weak counterexamples.

Which axioms does Brouwer actually use? He gives little or no justification for the conclusions drawn from the counterexamples, so I shall simply attribute to him the axioms that are needed for his conclusions to follow.

\subsection{Which Axioms are Really Needed?}

Axiom 0 is necessary for any of the arguments to work. In theorem $6 \mathrm{~b}$, for example, a rational sequence $a$ is defined by cases, with $a_{n}$ equal to a positive rational or 0 according to whether $\exists i \leq n\left(\vdash_{i} A \vee \vdash_{i} \neg A\right)$ holds. If this is to count as a welldefined sequence we must have $\forall n a_{n} \in \mathbb{Q}$, hence $\forall n\left(a_{n}>0 \vee a_{n}=0\right)$; hence 
$\exists i \leq n\left(\vdash_{i} A \vee \vdash_{i} \neg A\right)$ must be decidable; hence $\vdash_{i} A$ must be decidable. We need decidability again to show that $a$ is Cauchy. Axiom 0 is therefore indispensable.

Beyond that, the possible choices of axioms are

- $\quad$ axioms 1 and 2,

- axioms 1 and $2^{\prime}$,

- $\quad$ axioms $1^{\prime}$ and 2 (or equivalently 2 and 3 ),

- $\quad$ axioms $1^{\prime}$ and $2^{\prime}$ (or equivalently $2^{\prime}$ and 3 ).

Axiom 2 is only ever used in theorem $11 \mathrm{~b}$ and theorem $11 \mathrm{c}$. Even here, however, the desired weak counterexample to $x \notin \mathbb{Q} \vee x \notin \mathbb{Q}$ can be obtained using axiom $2^{\prime}$ instead; the desired weak counterexample to $x \notin \mathbb{Q} \Rightarrow x \in \mathbb{Q}$ could be obtained from theorem $6 \mathrm{~b}$ instead. One can accomplish all the arguments using axioms $1^{\prime}$ and $2^{\prime}$, provided one is prepared to accept a slightly weaker conclusion, i.e., $\neg A \vee \neg \neg A$ instead of $A \vee \neg A$.

\subsection{Axiom 1' or Axiom 2'?}

There is one case where there is a choice between using axiom 1 (or $1^{\prime}$ ) and using axiom $2^{\prime}$. In an example in [13], which is my theorem $7 \mathrm{~b}$, Brouwer uses axioms $2^{\prime}, 3$ to show

$$
\begin{aligned}
& x \not 0 \Rightarrow \neg x>0 \Rightarrow \neg \square A \Rightarrow \neg A \\
& x \not 0 \Rightarrow \neg x<0 \Rightarrow \neg \square \neg A \Rightarrow \neg \neg A .
\end{aligned}
$$

Thus if $x \notin 0 \vee x \# 0$ is generally true then so is $\neg A \vee \neg \neg A$.

Niekus [32, 33] asks why Brouwer doesn't use a more direct argument, using axiom 1 , giving

$$
\begin{aligned}
& x<0 \Rightarrow \square \neg A \Rightarrow \neg A \\
& x>0 \Rightarrow \square A \Rightarrow A
\end{aligned}
$$

Thus if $x<0 \vee x>0$ is generally true then so is $A \vee \neg A$. Niekus concludes that Brouwer does not want to use axiom 1.

Van Atten [39, p. 1618] objects that Niekus is confusing the two order relations, $x<0$ and $x \notin 0$. This seems to be true, but Niekus' argument can be easily repaired: just take the contrapositive of $x<0 \Rightarrow \neg A$ and $x>0 \Rightarrow A$ twice to give

$$
\begin{aligned}
& x \notin 0 \Rightarrow \neg A \\
& x \# 0 \Rightarrow \neg \neg A
\end{aligned}
$$

Thus if $x \notin 0 \vee x \# 0$ is generally true then so is $\neg A \vee \neg \neg A-$ the same conclusion as Brouwer reaches, by a different route. (Note that the argument can be stated using only axiom $1^{\prime}$, not axiom 1.)

These two routes from $x \notin 0 \vee x \not 0$ to $\neg A \vee \neg \neg A$ are theorem $7 \mathrm{~b}$ (vi,vii) above. Brouwer takes the route using axioms $2^{\prime}, 3$ rather than the route using axiom $1^{\prime}$. 
There is a more serious objection to Niekus' choice of axioms. He rejects axiom 1, $\square A \Rightarrow A$. He also rejects axiom $0, \vdash_{n} A \vee \nvdash_{n} A$, because he thinks it implies that the activity of the creative subject is lawlike. The axioms he does accept are

$$
\begin{aligned}
& \vdash_{n} A \Rightarrow \vdash_{n+m} A \\
& A \Rightarrow \square A \quad(\text { axiom 2) }
\end{aligned}
$$

These are too weak for any counterexample arguments. Without axiom 0 , the sequence defining the real number $x$ is ill-defined. Without axiom 1 or $1^{\prime}$ the theory admits a trivial interpretation in which $\vdash_{n} A$ holds for every $n$ and $A$. Axiom 2 or $2^{\prime}$ is no use without axiom 3 (this was why I introduced axiom 3). But axioms $2^{\prime}$ and 3 imply $1^{\prime}$, so assuming 2 or $2^{\prime}$ together with 3 is not really an alternative to assuming $1^{\prime}$.

\subsection{Axiom 1 or Axiom 1'?}

Does Brouwer use the strong axiom 1 or the weaker axiom $1^{\prime}$ ? There are several places where using axiom 1 would give a stronger conclusion than axiom 1 , i.e., $A \vee \neg A$ rather than $\neg A \vee \neg \neg A$. Brouwer's practice is variable.

When he begins the counterexample by saying 'Let $\alpha$ be an assertion which has neither been judged nor been recognised as judgeable', a use of axiom 1 is implied. This applies to [23]. In [11], in the density-in-itself and separability-in-itself arguments, a use of axiom 1 also seems to be implied.

When he begins by saying 'Let $\alpha$ be a mathematical assertion so far neither tested nor recognised as testable', a use of axiom $1^{\prime}$ is implied. This applies to the checkingnumber examples in [14], [20], [41, p. 50], and to the refutation of the B form of the Bolzano-Weierstrass theorem in [18, p. 517].

\section{What Added Value Does the Creative Subject Provide?}

All the weak counterexamples can be accomplished with or without use of the creative subject.

Weak counterexamples without the creative subject always work by showing that the proposition in question implies one of the formulae,

(i) $\exists n P(n) \vee \neg \exists n P(n)$,

(ii) $\neg \exists n P(n) \vee \neg \neg \exists n P(n)$,

(iii) $\neg \neg \exists n P(n) \Rightarrow \exists n P(n)$,

(iv) $\neg \exists n P^{\prime}(2 n) \vee \neg \exists n P^{\prime}(2 n+1)$,

(v) $\neg \neg \exists n P(n) \Rightarrow \neg \exists n P^{\prime}(2 n) \vee \neg \exists n P^{\prime}(2 n+1)$

(with the exception of theorem $14 \mathrm{a}$ (ii,iii)). In the corresponding version of the weak counterexample with the creative subject, the conclusion (i) or (iii) is replaced by $A \vee \neg A$ or $\neg \neg A \vee \neg A$ (depending on whether we assume axiom 1 or $1^{\prime}$ ). The other three conclusions are always replaced by $\neg \neg A \vee \neg A$. The creative subject allows us to construct a weak counterexample to (iii), i.e., a property $P$ for which we 
can prove $\neg \neg \exists n P(n)$ without being able to prove $\exists n P(n)$ (see theorem 4). Hence it provides a rationale for rejecting Markov's principle, provided we have rejected the principle of excluded middle.

Brouwer sometimes uses the creative subject and sometimes does not. For example, in [18] he uses a fleeing property (without the creative subject) to refute the A form of the Bolzano-Weierstrass theorem but uses the creative subject to refute the B form; he could just as well use either in each case. As a rule, Brouwer resorts to the creative subject in cases where the version without the creative subject would lead to the conclusion (iii) or (v). However, two exceptions to this are [41, p. 42, pp. 55-6]; see my theorem $7 \mathrm{a}$ and theorem $15 \mathrm{a}$.

Thus the creative subject gives a stronger conclusion. On the other hand, the version without the creative subject applies to the reduced continuum (consisting of the lawlike reals), and hence also to the full continuum (consisting of all reals), whereas the version with the creative subject applies only to the full continuum. In this respect, the version without the creative subject is stronger.

The essential function of a weak counterexample is to express explicitly and rigorously why a certain statement is not provable. Heyting states it in dialogue form:

INT. ... Nor is it important whether we call it a mathematical result or not. In any case it shows that it would be foolish to seek a prooffor the equivalence of the relations $\neq$ and \# between real numbers.

CLASS. I have been convinced of that since 2.2.3.

INT. Brouwer's example analyses one of the subconscious reasons which made you feel this conviction. [27, §8.1.1]

The weak counterexamples without the creative subject perform this function just as well as the versions with the creative subject.

\section{Arguments that go Beyond the Usual Theory of the Creative Subject}

\section{1 'Truth of $A$ and Rationality of the Conditional-Checking Number $x$ are Equivalent'}

Brouwer has two, possibly related, claims that fall outside the methods used above; in attempting to justify them we may get a deeper insight into the nature of the creative subject, the role of time in the argument, and possibly the connection with the theory of choice sequences.

In 1954 Brouwer considered the conditional checking-number $x$ of a drift $(a, u)$ through a proposition $A$, where $a \notin \mathbb{Q}$ and $\forall n u_{n} \in \mathbb{Q}$. He claimed that $A \Leftrightarrow x \in \mathbb{Q}$. As can be seen from my theorem $11 \mathrm{~b}$, this goes slightly beyond what I believe can be justified.

Let $A$ be a mathematical assertion so far neither tested nor recognized as testable. Then, in connection with the assertion $A$ and with a drift $(a, u)$ the creating subject can generate an infinitely proceeding sequence $s$ of limiting 
number cores $s_{1}, s_{2}, \ldots$ according to the following direction: As long as during the choice of the $s_{n}$ the creating subject has not experienced the truth of $A$ ..., each $s_{n}$ is chosen equal to a. But as soon as between the choice of $s_{r-1}$ and that of $s_{r}$ the creating subject has experienced the truth of $A \ldots, s_{r}$, and likewise $s_{r+v}$ for each natural number $v$, is chosen equal to $u_{r}$. This sequence s converges to a limiting number core $x \ldots$ which will be called a conditional checking-core of $(a, u)$ through A ...

Let $(a, u)$ be a drift whose counting cores $\left[u_{n}\right]$ are rational and whose kernel [a] is irrational. ...

... truth of $A$ and rationality of $x$ are equivalent. So the assertion of the rationality of $x$ is neither judgeable nor testable. ([20, p. 525], with notation altered)

(There are two similar cases in theorem 11c [14, p. 492] [20, p. 525] and theorem 13 b [14, p. 492]. Brouwer claims to show that $x \notin \mathbb{Q}$ does not imply $x \in \mathbb{Q}$. He needs a result of the form $x \in \mathbb{Q} \Rightarrow A \vee \neg A$ or $x \in \mathbb{Q} \Rightarrow A$ to complete the argument.)

Brouwer gave no justification for his claim that $A \Leftrightarrow x \in \mathbb{Q}$. Van Atten provides a plausible reconstruction of Brouwer's thinking:

Let $A$ be a proposition that is at present untestable, and $(a, u)$ a drift with rational numbers $u_{n}$ and an irrational $a$. Then truth of $A$ and rationality of the conditional checking-number $x$ are equivalent: the choices in $x$ become a fixed rational as soon as a construction for $A$ has been found, and only then. So long as the proposition $A$ is not testable, the proposition $x \in \mathbb{Q}$ is not testable, and, as decidability implies testability, the proposition $x \in \mathbb{Q} \vee x \notin \mathbb{Q}$ is not provable. ([39, §3.7], with notation altered)

The delicate point here is the phrase 'and only then'. I have been unable to convert this into a convincing proof. We cannot, simply by examining the available knowledge about how $x$ is generated, read off all its consequences. To assert that if $x \in \mathbb{Q}$ is provable then $A$ is provable, we would have to survey all possible proofs of $x \in \mathbb{Q}$. By this I mean that we would have to be able to say something about the general form that a proof of $x \in \mathbb{Q}$ must take, and thereby show that it must contain, or be transformable into, a proof of $A$. I can see no way of doing this.

Instead I provide a weak counterexample to show that $x \in \mathbb{Q}$ does not in general imply $A$, if $x$ is a conditional checking-number (the converse is theorem $11 \mathrm{~b}(\mathrm{i})$ ). As usual, I want to isolate the contribution of the creative subject, so I shall state it first for an arbitrary decidable property $P$ of natural numbers.

Def" . For any decidable property $P$, Brouwer's claim for $P, B C_{P}$, is the following statement.

For any drift $(a, u)$, with $\forall n u_{n} \# a$ and $\forall m, n\left(m \neq n \Rightarrow u_{m} \# u_{n}\right)$ and $a \notin \mathbb{Q}$ and $\forall n u_{n} \in \mathbb{Q}$, with checking-number $x$ of $(a, u)$ through $P$,

$$
x \in \mathbb{Q} \Rightarrow \exists n P(n) .
$$


Theorem 29a. For any decidable property $P$ of natural numbers, if $B C_{P}$ holds then $\neg \neg \exists n P(n) \Rightarrow \exists n P(n)$.

Proof Consider any decidable property $P$ of natural numbers.

Suppose $B C_{P}$.

Suppose $\neg \neg \exists n P(n)$.

Choose a strictly increasing rational sequence $u$ converging to an irrational $a$; e.g., choose $a=\sqrt{2}$ and $u=(1,1.4,1.41,1.414, \ldots)$, skipping repetitions. Note that this gives a drift $(a, u)$ with the required conditions, $\forall n u_{n} \# a$ and $\forall m, n\left(m \neq n \Rightarrow u_{m} \# u_{n}\right)$.

Construct the checking-number $x$ of $(a, u)$ through $P$.

Define a new drift $(b, v)$ by $b=a-x$ and $\forall n v_{n}=u_{n}-x$.

Suppose $b \in \mathbb{Q}$.

Then, since $a \notin \mathbb{Q}$, this implies $x=a-b \notin \mathbb{Q}$,

so by theorem 11 a(ii) $\neg \exists n P(n)$, contrary to the hypothesis $\neg \neg \exists n P(n)$.

This shows that $b \notin \mathbb{Q}$. (1)

Note that $\forall n v_{n}<b$ and $\forall n v_{n}<v_{n+1}$.

Define a further sequence $w$ of reals as follows. For any $n$, if $\exists i \leq n P(i)$ then define $w_{n}=v_{n}$; otherwise choose $w_{n}$ as a rational number between $v_{n}$ and $v_{n+1}$ (this choice is made at stage $n$ ).

Then $\forall n v_{n} \leq w_{n}<v_{n+1} \leq w_{n+1}<v_{n+2}<b$.

This gives the conditions $\forall n w_{n} \# b$ and $\forall m, n\left(m \neq n \Rightarrow w_{m} \# w_{n}\right)$. (2)

Consider any $n$.

Suppose $\exists i \leq n P(i)$.

Choose the least $r$ such that $P(r)$.

Then $r \leq n$ and $P^{\prime}(r)$,

so, by definition of a checking-number, $x=u_{r}$,

so $w_{n}=v_{n}=u_{n}-u_{r} \in \mathbb{Q}$.

This shows that $\exists i \leq n P(i) \Rightarrow w_{n} \in \mathbb{Q}$.

Also, by construction, we have $\neg \exists i \leq n P(i) \Rightarrow w_{n} \in \mathbb{Q}$.

So in both cases $w_{n} \in \mathbb{Q}$.

This shows that $\forall n w_{n} \in \mathbb{Q}$. (3)

For any $n,\left|w_{n}-b\right| \leq\left|v_{n}-b\right|=\left|u_{n}-a\right|$,

and hence $w_{n} \rightarrow b$ as $n \rightarrow \infty$.

Thus $(b, w)$ is a drift. (4)

Construct its checking-number $y$ through $P$.

Suppose $\exists n P(n)$.

Choose the least $r$ such that $P(r)$.

Then, by definition of a checking-number, $x=u_{r}$ and $y=w_{r}$.

So $y=w_{r}=v_{r}=u_{r}-x=0$.

This shows that $\exists n P(n) \Rightarrow y=0$. 
Contraposing this twice gives $\neg \neg \exists n P(n) \Rightarrow y=0$.

Since we have $\neg \neg \exists n P(n)$ by hypothesis, this gives $y=0$.

Hence $y \in \mathbb{Q}$.

By (1), (2), (3) and (4) we can apply $B C_{P}$ to $(b, w)$, giving $\exists n P(n)$.

This shows that $\neg \neg \exists n P(n) \Rightarrow \exists n P(n)$.

This shows that if $B C_{P}$ then $\neg \neg \exists n P(n) \Rightarrow \exists n P(n)$.

This proves the theorem.

The theorem shows that any general proof of $B C_{P}$ (for all $P$ or for a class of $P$ s) would give a proof of Markov's principle $\neg \neg \exists n P(n) \Rightarrow \exists n P(n)$ for that class of $P$ s.

This theorem is applied to the creative subject as follows.

Def" . For any proposition A, Brouwer's claim for $A, B C_{A}$, is the following statement.

For any drift $(a, u)$, with $\forall n u_{n} \# a$ and $\forall m, n\left(m \neq n \Rightarrow u_{m} \# u_{n}\right)$ and $a \notin \mathbb{Q}$ and $\forall n u_{n} \in \mathbb{Q}$, with conditional checking-number $x$ of $(a, u)$ through $A$,

$$
x \in \mathbb{Q} \Rightarrow A .
$$

Theorem 29b. For any proposition $A$, if $B C_{A}$ holds then $\neg \neg A \Rightarrow A$. [axioms 1,2]

Proof Consider any proposition $A$.

Define the conditional property $P$ of $A$. (This gives $\exists n P(n) \Leftrightarrow \square A$.)

By axiom $2, x \in \mathbb{Q} \Rightarrow A$ implies $x \in \mathbb{Q} \Rightarrow \square A$, i.e., $x \in \mathbb{Q} \Rightarrow \exists n P(n)$.

So

$$
\begin{array}{rlrl}
B C_{A} & \Rightarrow B C_{P} & \\
& \Rightarrow(\neg \neg \square A \Rightarrow \square A) & & \text { by theorem } 29 \mathrm{a} \\
& \Rightarrow(\neg \neg A \Rightarrow A) & & \text { by axioms } 1,2^{\prime} .
\end{array}
$$

This proves the theorem.

This theorem shows that if Brouwer's claim were generally true then so would be $\neg \neg A \Rightarrow A$, and hence the principle of excluded middle would be valid. We cannot therefore assert $B C_{A}$ for all $A$.

However, Mark van Atten (personal communication) has raised several objections to my formulation of the argument.

1. Van Atten proposes that $B C_{A}$ should be stated not as an implication $(x \in \mathbb{Q} \Rightarrow$ $A$ ) but as a rule of proof (If $x \in \mathbb{Q}$, and all the other conditions, can be proved without hypotheses then $A$ can be proved without hypotheses); see [35, S2] for the significance of this distinction. $B C_{P}$ would be similarly reworded. The proofs of theorems $29 \mathrm{a}$ and $29 \mathrm{~b}$ go through with slight changes. Theorem 29a now says: for any decidable property $P$ of natural numbers for which $B C_{P}$ and $\neg \neg \exists n P(n)$ hold, $\exists n P(n)$ holds. Theorem $29 \mathrm{~b}$ now says: for any proposition $A$ 
for which $B C_{A}$ and $\neg \neg A$ hold, $A$ holds. By taking $A$ as $B \vee \neg B$, for arbitrary $B$, we still obtain a proof of the principle of excluded middle.

2. Van Atten points out that Brouwer's argument uses an untestable proposition $A$, whereas my counterexample uses a testable $A$ (namely $B \vee \neg B$ ); I therefore seem to have taken 'Brouwer's claim' out of its original context. It might be thought, therefore, (though this is not van Atten's opinion) that $B C_{A}$ should be asserted for all untestable $A$, rather than for all $A$. It is, however, hard to see a rationale for this. Consider the situation again. We have a formula $A$ for which, at time $t_{0}$ (now), we lack a proof of $\neg A$ or $\neg \neg A$. At some future time, $t_{1}$, we may find a proof of $x \in \mathbb{Q}$. Brouwer's claim is that this is only possible if we have a proof of $A$ at time $t_{1}$ : that is, we can prove $x \in \mathbb{Q}$ only via $A$. Why should the plausibility of this claim about time $t_{1}$ be dependent on our lack of a proof of $\neg A$ or $\neg \neg A$ at time $t_{0}$ ? If it is plausible for currently untestable $A$ then surely it is plausible for all $A$.

3. The conditional checking-number $x$ is given as the limit of an infinite sequence; but the timing of the 'stages' at which the terms of the sequence are chosen may affect the value of $x$. Up until now I have thought of these stages as given by a predetermined schedule; van Atten, however, proposes that the creative subject should choose the terms at freely chosen times, unless it decides to make these times dependent on another mathematical development (as in Brouwer [15, p496] and [22, p543]). If so, there will be many possible conditional checking-numbers for a given drift and proposition A. Brouwer's 'direction' (quoted above from [20, p525]) for choosing the sequence does not constrain the timings of the stages; and he does indeed speak of 'a', rather than 'the', conditional checking-number. If this is right, I need to reword $B C_{A}$ to read: for any drift $(a, u) \ldots$, and for any conditional checking-number $x$ of $(a, u)$ through $A$, $\ldots$. The proof of theorem $29 \mathrm{~b}$ still goes through, and the same conclusion is reached. Note that $B C_{P}$ and theorem $29 \mathrm{a}$ are not affected. The important point is that the stages of the sequence determining $y$ coincide in time with the stages of the sequence determining $x$. I am assuming here that the creative subject, when choosing the sequence determining $y$, is allowed to adopt a rule determining the future timings to agree with those of $x$. Van Atten's view is (1) that the creative subject clearly has the freedom to construct, and theorise about, sequences that are subject to a certain set of conditions and no others; (2) that the 'direction' Brouwer gives [20, p525] is an example of the exercise of that freedom; (3) that, thus understood, and given Brouwer's conception of truth, Brouwer's claim is correct. In my view, since Brouwer's direction makes no stipulation about the timing of the choices of the terms, the creative subject is free to adopt a rule on the timings, without violating the definition of a conditional checking-number.

\subsection{The Negative Continuity Proof of 1927}

The second of Brouwer's claims that goes beyond the usual theory of the creative subject is the 1927 theorem in [8] that all functions are negatively continuous (see the definition of negative continuity above, just before theorem 19a). Here is the argument in full. 
Theorem 1 Every full function is negatively continuous.

Proof Let $f(x)$ be a full function, let $\xi_{0}$ be an arbitrary point core $x$, and let $\xi_{1}, \xi_{2}$, . . be a fundamental sequence of point cores $x$ that converges positively to $\xi_{0}$. We now assume for the moment that there exist a natural number $p$ and a fundamental sequence $p_{1}, p_{2}, \ldots$ of monotonically increasing natural numbers such that $\left|f\left(\xi_{p_{v}}\right)-f\left(\xi_{0}\right)\right|>1 / p$ for every $v$, and we define a point core $\xi_{\omega}$ of the unit continuum by starting from an unlimited sequence $F_{1}$ of generating intervals of a point belonging to $\xi_{0}$ and then constructing, by means of an unlimited sequence of choices of $\lambda$-intervals, a point $F_{2}$ of the unit continuum in such a way that we temporarily choose, for every natural number $n$ that we have already considered, the first $n$ intervals identical with the first $n$ intervals of $F_{1}$ but reserve the right to determine, at any time after the first, second,..., $(m-1)$ th, and mth intervals have been chosen, the choice of all further intervals (that is, of the $(m+1) t h,(m+2) t h$, and so on) in such a way that either a point belonging to $\xi_{0}$ or one belonging to a certain $\xi_{p_{v}}$ is generated. Then the function $f(x)$ is not defined for the point core $\xi_{\omega}$ containing $F_{2}$; this brings us to a contradiction, and our assumption has proved to be illegitimate. But this means that the function $f(x)$ is negatively continuous. [8, p459]

This is said to be 'an immediate consequence of the intuitionistic point of view': this means that it is not dependent on well-orderings and bar induction, as the betterknown uniform continuity theorem is.

Different explanations of this proof are given in the literature [31, 34, 37, 43, 44] [39, appendix B]. Niekus [32] comments that Brouwer's proof 'is vague and deviates essentially from all his other uses of choice sequences' (p. 32), and also 'This proof of the negative continuity theorem is so vague that it allows many reconstructions. ... But in no way can this sequence be called characteristic for Brouwer's use of individual choice sequences' (p. 40). Indeed. That is what makes it so interesting.

The proof turns on the notion of a choice sequence as an object with a fixed intensional identity, even when only a few of its terms are determined. I shall give two reconstructions of the proof, one using the creative subject and the other using Appleby's [1] theory of knowledge states. In the conventional view a choice sequence $x$ is seen as a generating process, an 'incomplete object' that grows over time as its terms $x_{0}, x_{1}, x_{2}, \ldots$ are successively chosen. At the same time as the terms are being chosen, restrictions may be chosen that limit the future choice of terms. A restriction may take the form of a spread, constraining the future terms, or perhaps a law, determining all terms from now on, or a law-relation, by which the sequence is constrained in a lawlike way relative to another sequence. New facts about $x$ become true as more and more terms and restrictions are chosen and as we find more and more proofs. Any conclusion $A(x)$ we draw about $x$ must be proved at a particular time (by the axiom $A(x) \Rightarrow \square A(x)$ ), and so must be based on the terms and restrictions that have been chosen up to that time, and once proved it must continue to hold regardless of how $x$ develops in future.

In Appleby's view, in contrast, a sequence $x$ is not a growing object but a 'black box' that produces any term on demand. We may query it by asking ' $n$ ?' and it 
must reply with $x_{n}$. It may also volunteer intensional information about itself, such as a spread, a law or a law-relation, at any time. All the information it provides must be consistent. We never know whether there is more intensional information still to come. Any conclusion $A(x)$ we draw about $x$ must be proved; a proof is a finite construction and so must contain a finite amount of information about $x$. This information is expressed as a knowledge state: for example, $S E(n, m)$ is the knowledge that the $n$th term is $m$; Spread $(s)$ is the knowledge that the sequence must conform to a spread $s ; \operatorname{Law}(f)$ is the knowledge that the sequence must obey a law $f$. A general knowledge state is built up out of atomic knowledge states like these by finite conjunction and disjunction. If $A(x)$ holds then this must be because $x$ satisfies some knowledge state.

In the light of this it seems to follow that, when a function $f$ is applied to a sequence $x$, giving a natural number value, the computation may be viewed as a dialogue or two-person game between $f$ and $x$, as suggested by van Atten $\&$ van Dalen [40]: $f$ queries $x$ for particular terms, and $x$ answers; $x$ may volunteer intensional information about itself; eventually, after a finite number of moves, $f$ must announce the value $f(x)$; and once it has done so it cannot retract its answer, whatever $x$ 's future behaviour. (In the case where $f$ maps $x$ to a real number, it does not need to determine $f(x)$ exactly but must eventually determine $f(x)$ to any desired degree of precision.)

Theorem 30 (negative continuity theorem in [8]). Any function $f: \mathbb{R} \rightarrow \mathbb{R}$ is negatively continuous.

Proof We shall use the binary representation of real numbers $(\$ 2)$.

Consider any function $f: \mathbb{R} \rightarrow \mathbb{R}$.

Consider any point of positive discontinuity $a$ of $f$.

Choose a sequence $u$ of real numbers and a rational $\epsilon>0$ such that $u_{n} \rightarrow a$ as $n \rightarrow \infty$ and $\forall n\left|f\left(u_{n}\right)-f(a)\right| \geq \epsilon$.

For the drift $(a, u)$, choose the function $v: \mathbb{N} \rightarrow \mathbb{N}$, the real $a^{\prime}$ and the sequence $u^{\prime}$ of reals as in theorem 9 .

Note that since $0<\epsilon$, we have $\forall y \in \mathbb{R}(0<|y-f(a)| \vee|y-f(a)|<\epsilon)$.

We seek a contradiction.

(Version of the proof using the creative subject)

Consider a real number $x$ constructed as a choice sequence of integers as follows: the chooser imposes an initial restriction that $x$ belong to the spread $\mathbb{R}$, and then chooses terms $x_{0}=a_{0}^{\prime}, x_{1}=a_{1}^{\prime}, x_{2}=a_{2}^{\prime}, \ldots$, as a sequence of individual choices, without imposing any further restrictions.

Since $f$ is total, there exists a stage $n$ after which $f(x)$ is determined to any desired degree of precision, in particular it is determined whether $0<\mid f(x)-$ $f(a) \mid$ or $|f(x)-f(a)|<\epsilon$.

(Note: I am not saying that all terms of $x$ are chosen to agree with $a^{\prime}$, merely that this is done until $f$ delivers a verdict on whether $0<|f(x)-f(a)|$ or $|f(x)-f(a)|<\epsilon$, which $f$ must do eventually.) 
Thus there exists $n$ such that either every continuation of $x$ after $x_{0}=a_{0}^{\prime}, \ldots x_{n}=a_{n}^{\prime}$ gives $0<|f(x)-f(a)|$, or every continuation gives $|f(x)-f(a)|<\epsilon$.

Now, at the next stage, the chooser may adopt a law-relation that all the terms of $x$ shall equal the terms of $a^{\prime}$ from now on.

This would give $x=a^{\prime}=a$ (i.e., they are equal as real numbers), so $f(x)=f(a)$, so $|f(x)-f(a)|=0$.

Alternatively, the chooser may adopt a law-relation at the next stage that all the terms of $x$ shall equal the terms of $u_{r}^{\prime}$ from now on, for $r=v(n)+1$, giving $\forall i x_{i}=\left\{\begin{array}{ll}a_{i}^{\prime} & \text { if } i \leq n \\ u_{r i}^{\prime} & \text { if } i>n\end{array}\right.$, which gives $x \in \mathbb{R}$ by theorem 9(iii).

This would give $x=u_{r}^{\prime}=u_{r}$ (they are equal as real numbers), so $f(x)=f\left(u_{r}\right)$, so $|f(x)-f(a)| \geq \epsilon$.

This is the desired contradiction.

(Version of the proof using knowledge states)

Consider a real number $x$ constructed as a choice sequence of integers as follows: $x$ volunteers the intensional information that it belongs to the spread $\mathbb{R}$, and then replies to any query ' $i$ ?' with $a_{i}^{\prime}$, without volunteering any further intensional information.

Since $f$ is total it must eventually determine $f(x)$ to any desired degree of precision, in particular it announces whether $0<|f(x)-f(a)|$ or

$|f(x)-f(a)|<\epsilon$, after a finite number of queries ' $i_{1}$ ?', $\ldots$ ' $i_{k}$ ?'.

Choose $n=\max \left(i_{1}, \ldots i_{k}\right)$.

$x$ may then volunteer the information that it satisfies the law-relation

$\forall i x_{i}=a_{i}^{\prime}$.

In that case $x=a^{\prime}=a$, and hence $f(x)=f(a)$, so $|f(x)-f(a)|=0$.

Alternatively, $x$ may volunteer the law-relation that

$$
\forall i x_{i}= \begin{cases}a_{i}^{\prime} & \text { if } i \leq n \\ u_{r i}^{\prime} & \text { if } i>n\end{cases}
$$

for $r=v(n)+1$,

which gives $x=u_{r}^{\prime}=u_{r}$, so $f(x)=f\left(u_{r}\right)$, so $|f(x)-f(a)| \geq \epsilon$.

One of these will contradict the prior decision on whether $0<|f(x)-f(a)|$ or $|f(x)-f(a)|<\epsilon$.

This is the desired contradiction.

(The two versions rejoin here.)

This shows that $f$ has no point of positive discontinuity, i.e., $f$ is negatively continuous.

This shows that any function $f: \mathbb{R} \rightarrow \mathbb{R}$ is negatively continuous.

Notice that, if the trick used in the proof is valid (as I think it is), it can equally be used to prove that all functions are positively continuous, i.e., continuous in the usual (pointwise) sense. It can be further generalised to prove the weak continuity principle for numbers (WC-N), a fundamental axiom of intuitionistic analysis whose justification is still controversial [40]. 


\section{Theorem 31 (WC-N).}

$\forall \alpha \exists m A(\alpha, m) \Rightarrow \forall \alpha \exists m, n \forall \beta(\bar{\alpha}(n)=\bar{\beta}(n) \Rightarrow A(\beta, m))$

where $\alpha, \beta$ range over infinite sequences of natural numbers, $\bar{\alpha}(n)=$ $\left\langle\alpha_{0}, \alpha_{1}, \ldots \alpha_{n-1}\right\rangle$, and the formula $A(\alpha, m)$ is extensional in $\alpha$, i.e.,

$$
\forall \alpha, \beta \forall m(A(\alpha, m) \wedge \alpha=\beta \Rightarrow A(\beta, m))
$$

where $\alpha=\beta$ means $\forall i \alpha_{i}=\beta_{i}$.

Proof (Version using the creative subject)

Suppose $\forall \alpha \exists m A(\alpha, m)$.

Consider any sequence $\alpha$.

Consider a sequence $\gamma$ constructed as follows: the chooser chooses its terms $\gamma_{0}=\alpha_{0}, \gamma_{1}=\alpha_{1}, \gamma_{2}=\alpha_{2}, \ldots$, as a sequence of individual choices, without imposing any restrictions.

This continues until a number $m$ is determined such that $A(\gamma, m)$. This must happen eventually, after some number of choices $\gamma_{0}=\alpha_{0}, \ldots \gamma_{n-1}=\alpha_{n-1}$, since $\forall \alpha \exists m A(\alpha, m)$.

Whatever further choices (of terms or restrictions) are made for $\gamma$, it will continue to be the case that $A(\gamma, m)$.

Consider any sequence $\beta$ such that $\bar{\alpha}(n)=\bar{\beta}(n)$.

Therefore $\bar{\gamma}(n)=\bar{\beta}(n)$.

At the next stage, the chooser may adopt a law-relation that

$\forall i \geq n \gamma(i)=\beta(i)$.

In that case, $\gamma=\beta$, so, since $A$ is extensional, $A(\beta, m)$.

This shows that $\forall \beta(\bar{\alpha}(n)=\bar{\beta}(n) \Rightarrow A(\beta, m))$.

Thus $\exists m, n \forall \beta(\bar{\alpha}(n)=\bar{\beta}(n) \Rightarrow A(\beta, m))$.

This shows that $\forall \alpha \exists m, n \forall \beta(\bar{\alpha}(n)=\bar{\beta}(n) \Rightarrow A(\beta, m))$.

This shows that $\forall \alpha \exists n A(\alpha, n) \Rightarrow \forall \alpha \exists m, n \forall \beta(\bar{\alpha}(m)=\bar{\beta}(m) \Rightarrow A(\beta, n))$, as required.

(Version using knowledge states)

Suppose $\forall \alpha \exists m A(\alpha, m)$.

Then there is a function $f$ mapping sequences to natural numbers such that $\forall \alpha A(\alpha, f(\alpha))$. (Note that $f$ may not be extensional.)

Consider any sequence $\alpha$.

Consider a sequence $\gamma$ that behaves as follows: $\gamma$ responds to any query ' $i$ ?' by replying $\alpha_{i}$, without volunteering any intensional information. It does this as long as no value $f(\gamma)$ is announced.

$f$ must eventually announce a value $f(\gamma)$, after a number of queries ' $i_{1}$ ?', $\ldots$ '. 'i $i_{k}$ ?'.

Then $A(\gamma, m)$ holds, where $m=f(\gamma)$.

Choose $n=\max \left(i_{1}, \ldots i_{k}\right)+1$. 
Consider any sequence $\beta$ such that $\bar{\alpha}(n)=\bar{\beta}(n)$.

Therefore $\beta_{i_{1}}=\alpha_{i_{1}}=\gamma_{i_{1}}, \ldots, \beta_{i_{k}}=\alpha_{i_{k}}=\gamma_{i_{k}}$.

We are committed to $A(\gamma, m)$, whatever $\gamma$ 's subsequent behaviour.

$\gamma$ may now volunteer the law-relation that $\forall i \gamma(i)=\beta(i)$.

We thus have $\gamma=\beta$, so, since $A$ is extensional, $A(\beta, m)$.

This shows that $\forall \beta(\bar{\alpha}(n)=\bar{\beta}(n) \Rightarrow A(\beta, m))$.

Thus $\exists m, n \forall \beta(\bar{\alpha}(n)=\bar{\beta}(n) \Rightarrow A(\beta, m))$.

This shows that $\forall \alpha \exists m, n \forall \beta(\bar{\alpha}(n)=\bar{\beta}(n) \Rightarrow A(\beta, m))$.

This shows that $\forall \alpha \exists n A(\alpha, n) \Rightarrow \forall \alpha \exists m, n \forall \beta(\bar{\alpha}(m)=\bar{\beta}(m) \Rightarrow A(\beta, n))$, as required.

Thus the negative continuity proof may be seen as a special case of a WC-N proof. Indeed, Veldman [44] assimilates Brouwer's negative continuity proof to an application of WC-N and shows that WC-N leads to positive continuity (theorem 2.6) and also to strong counterexamples that go far beyond Brouwer's claims at that point. This works well mathematically but raises a difficult exegetic question of why Brouwer did not state his theorem for positive continuity or draw any connection with his previous uses of continuity principles similar to WC-N in [4] and [7].

Where I disagree with Veldman is that he treats the negative continuity theorem as a weak counterexample, using a fleeing property (theorem 3.2). This is not how Brouwer stated it. Many years later, in his Cambridge lectures, Brouwer gave a completely different proof of the same theorem using a weak counterexample (see theorem 19a above). As van Atten [39, appendix B] remarks, the fact that Brouwer presented a new proof is no indication that he had come to have second thoughts about his 1927 proof.

\section{Conclusions}

1. Weak counterexamples are genuine mathematical proofs.

2. The creative subject is not really necessary for the weak counterexamples. If it is used, the axioms $0\left(\vdash_{n} A \vee \nvdash_{n} A\right), 1^{\prime}(\neg A \Rightarrow \neg \square A)$ and $2^{\prime}(\neg \square A \Rightarrow \neg A)$ will suffice.

3. Brouwer's 1927 negative continuity theorem is a powerful example of the use of the concept of choice sequence, but it is not a weak counterexample and does not need the creative subject.

4. All of the theorems presented in this paper can be stated and proved without dependence on subjectivity or contingency. Infinite sequences need not be considered as 'incomplete objects' that grow over time; time is involved only in the innocuous sense that the application of a function to a choice sequence is seen as a finite sequence of computation steps.

Acknowledgements Thanks to James Appleby and Mark van Atten for helpful discussions, particularly in relation to $\S 9$. The conclusions drawn in this paper are my own responsibility. 
Open Access This article is licensed under a Creative Commons Attribution 4.0 International License, which permits use, sharing, adaptation, distribution and reproduction in any medium or format, as long as you give appropriate credit to the original author(s) and the source, provide a link to the Creative Commons licence, and indicate if changes were made. The images or other third party material in this article are included in the article's Creative Commons licence, unless indicated otherwise in a credit line to the material. If material is not included in the article's Creative Commons licence and your intended use is not permitted by statutory regulation or exceeds the permitted use, you will need to obtain permission directly from the copyright holder. To view a copy of this licence, visit http://creativecommonshorg/licenses/by/4.0/.

\section{References}

1. Appleby, J.F. (2017). Choice sequences and knowledge states: Extending the notion of finite information to produce a clearer foundation for intuitionistic analysis. PhD thesis, Keele University. http:// eprints.keele.ac.uk/4359/.

2. Bridges, D., \& Richman, F. (1987). Varieties of constructive mathematics. Cambridge: Cambridge University Press.

3. Brouwer, L.E.J. (1907). On the foundations of mathematics. PhD thesis, University of Amsterdam. In Heyting, A. (Ed.) L.E.J. Brouwer: Collected Works, vol. 1, Philosophy and foundations of mathematics (pp. 13-101). Amsterdam: North-Holland.

4. Brouwer, L.E.J. (1918). Begründung der Mengenlehre unabhängig vom logischen Satz vom ausgeschlossenen Dritten. Erster Teil: Allgemeine Mengenlehre. In Heyting, A. (Ed.) L.E.J. Brouwer: Collected works, vol. 1, Philosophy and foundations of mathematics (pp. 150-190). Amsterdam: North-Holland.

5. Brouwer, L.E.J. (1923). On the significance of the principle of excluded middle in mathematics, especially in function theory. In van Heijenoort, J. (Ed.) From Frege to Gödel: A source book in mathematical logic, 1879-1931 (pp. 334-341). Cambridge: Harvard University Press.

6. Brouwer, L.E.J. (1923). Intuitionistiche Zerlegung mathematischer Grundbegriffe. In Heyting, A. (Ed.) L.E.J. Brouwer: Collected Works, vol. 1, Philosophy and foundations of mathematics (pp. 275-280). Amsterdam: North-Holland.

7. Brouwer, L.E.J. (1925). Zur Begründung der intuitionistischen Mathematik I. In Heyting, A. (Ed.) L.E.J. Brouwer: Collected Works, vol. 1, Philosophy and foundations of mathematics (pp. 301-314). Amsterdam: North-Holland.

8. Brouwer, L.E.J. (1927). On the domains of definition of functions. In van Heijenoort, J. (Ed.) From Frege to Gödel: A source book in mathematical logic, 1879-1931 (pp. 446-463). Cambridge: Harvard University Press.

9. Brouwer, L.E.J. (1928). Intuitionistic reflections on formalism. In Mancosu, P. (Ed.) From Brouwer to Hilbert: The debate on the foundations on mathematics in the 1920s (pp. 40-44). New York: Oxford University Press.

10. Brouwer, L.E.J. (1929). Mathematik, Wissenschaft und Sprache. In Heyting, A. (Ed.) L.E.J. Brouwer: Collected works, vol. 1, Philosophy and foundations of mathematics (pp. 417-428). Amsterdam: North-Holland.

11. Brouwer, L.E.J. (1930). The structure of the continuum. In Mancosu, P. (Ed.) From Brouwer to Hilbert: The debate on the foundations on mathematics in the 1920s (pp. 54-63). New York: Oxford University Press.

12. Brouwer, L.E.J. (1933). Volition, knowledge, language, Section 3. In Heyting, A. (Ed.) L.E.J. Brouwer: Collected works, vol. 1, Philosophy and foundations of mathematics (pp. 443-446). Amsterdam: North-Holland.

13. Brouwer, L.E.J. (1948). Essentially negative properties. In Heyting, A. (Ed.) L.E.J. Brouwer: Collected works, vol. 1, Philosophy and foundations of mathematics (pp. 478-479). Amsterdam: North-Holland.

14. Brouwer, L.E.J. (1948). Consciousness, philosophy, and mathematics. In Heyting, A. (Ed.) L.E.J. Brouwer: Collected works, vol. 1, Philosophy and foundations of mathematics (pp. 480-494). Amsterdam: North-Holland.

15. Brouwer, L.E.J. (1949). The non-equivalence of the constructive and the negative order relation on the continuum. In Heyting, A. (Ed.) L.E.J. Brouwer: Collected works, vol. 1, Philosophy and foundations of mathematics (pp. 495-496). North-Holland: Amsterdam. 
16. Brouwer, L.E.J. (1950). Remarques sur la notion d'ordre. In Heyting, A. (Ed.) L.E.J. Brouwer: Collected works, vol. 1, Philosophy and foundations of mathematics (pp. 499-500). Amsterdam: North-Holland.

17. Brouwer, L.E.J. (1950). Sur la possibilité d'ordonner le continu. In Heyting, A. (Ed.) L.E.J. Brouwer: Collected works, vol. 1, Philosophy and foundations of mathematics (pp. 501-502). Amsterdam: North-Holland.

18. Brouwer, L.E.J. (1952). On accumulation cores of infinite core species. In Heyting, A. (Ed.) L.E.J. Brouwer: Collected works, vol. 1, Philosophy and foundations of mathematics (pp. 516-518). Amsterdam: North-Holland.

19. Brouwer, L.E.J. (1952). Fixed cores which cannot be found, though they are claimed to exist by classical theorems. In Heyting, A. (Ed.) L.E.J. Brouwer: Collected works, vol. 1, Philosophy and foundations of mathematics (pp. 519-521). Amsterdam: North-Holland.

20. Brouwer, L.E.J. (1954). Points and spaces. In Heyting, A. (Ed.) L.E.J. Brouwer: Collected works, vol. 1, Philosophy and foundations of mathematics (pp. 522-538). Amsterdam: North-Holland.

21. Brouwer, L.E.J. (1954). Addenda and corrigenda on the role of the principium tertii exclusi in mathematics. In van Heijenoort, J. (Ed.) From Frege to Gödel: A source book in mathematical logic, 1879-1931 (pp. 341-342). Cambridge: Harvard University Press.

22. Brouwer, L.E.J. (1954). Further addenda and corrigenda on the role of the principium tertii exclusi in mathematics. In van Heijenoort, J. (Ed.) From Frege to Gödel: A source book in mathematical logic, 1879-1931 (pp. 342-345). Cambridge: Harvard University Press.

23. Brouwer, L.E.J. (1954). Intuitionistic differentiability. In Heyting, A. (Ed.) L.E.J. Brouwer: Collected works, vol. 1, Philosophy and foundations of mathematics (pp. 546-548). Amsterdam: North-Holland.

24. Brouwer, L.E.J. (1954). An example of contradictority in classical theory of functions. In Heyting, A. (Ed.) L.E.J. Brouwer: Collected works, vol. 1, Philosophy and foundations of mathematics (pp. 549-550). Amsterdam: North-Holland.

25. Brouwer, L.E.J. (1955). The effect of intuitionism on classical algebra of logic. In Heyting, A. (Ed.) L.E.J. Brouwer: Collected works, vol. 1, Philosophy and foundations of mathematics (pp. 551-554). Amsterdam: North-Holland.

26. Dummett, M. (1977). Elements of intuitionism. Oxford: Oxford University Press.

27. Heyting, A. (1956). Intuitionism: An introduction. Amsterdam: North-Holland.

28. Ishihara, H. (1991). Continuity and nondiscontinuity in constructive mathematics. Journal of Symbolic Logic, 56(4), 1349-1354.

29. Kreisel, G. (1967). Informal rigour and completeness proofs. In Lakatos, I. (Ed.) Problems in the Philosophy of Mathematics (pp. 138-171). Amsterdam: North-Holland.

30. Martino, E. (1982). Creative subject and bar theorem. In Troelstra, A.S., \& van Dalen, D. (Eds.) The L.E.J. Brouwer centenary symposium (pp. 311-318). Amsterdam: North Holland.

31. Martino, E. (1985). On the Brouwerian concept of negative continuity. Journal of Philosophical Logic, 14, 379-398.

32. Niekus, J. (2010). Brouwer's incomplete objects. History and Philosophy of Logic, 31, 31-46.

33. Niekus, J. (2017). What is a choice sequence? How a solution to Troelstra's paradox shows the way to an answer to this question. Technical report PP-2017-02, Institute for Logic, Language and Computation, Amsterdam.

34. Posy, C. (1976). Varieties of indeterminacy in the theory of general choice sequences. Journal of Philosophical Logic, 5(1), 91-132.

35. Sundholm, G., \& van Atten, M. (2008). The proper explanation of intuitionistic logic: on Brouwer's demonstration of the Bar Theorem. In van Atten, M., Boldini, P., Bourdeau, M., Heinzmann, G. (Eds.) One Hundred Years of Intuitionism (1907-2007), The Cerisy Conference (pp. 60-77). Basel: Birkhäuser.

36. Troelstra, A.S. (1969). Principles of intuitionism. Lecture notes in mathematics Vol. 95. Berlin: Springer-Verlag.

37. Troelstra, A.S. (1982). On the origin and development of Brouwer's concept of choice sequence. In Troelstra, A.S., \& van Dalen, D. (Eds.) The L.E.J. Brouwer Centenary Symposium (pp. 465-486). Amsterdam: North Holland.

38. Troelstra, A.S., \& van Dalen, D. (1988). Constructivism in Mathematics Vol. I and II. Amsterdam: North Holland.

39. van Atten, M. (2018). The creating subject, the Brouwer-Kripke schema, and infinite proofs. Indagationes Mathematicae, 29, 1565-1636. 
40. van Atten, M., \& van Dalen, D. (2002). Arguments for the continuity principle. Bulletin of Symbolic Logic, 8(3), 329-347.

41. van Dalen, D. (Ed.) (1981). Brouwer's cambridge lectures on intuitionism. Cambridge: Cambridge University Press.

42. van Dalen, D. (ed.) (1992). L.E.J. Brouwer, Intuitionismus. Mannheim: Bibliographisches Institut, Wissenschaftsverlag.

43. Veldman, W. (1982). On the Continuity of Functions in Intuitionistic Real analysis: Some Remarks on Brouwer's Paper 'Über Definitionsbereiche von Funktionen'. Technical report 8210 Mathematisch Instituut, Katholieke Universiteit, Nijmegen.

44. Veldman, W. (2001). Understanding and using Brouwer's continuity principle. In Schuster, P., Berger, U., Osswald, H. (Eds.) Reuniting the antipodes - constructive and nonstandard views of the continuum (pp. 285-302). Synthese Library: Studies in Epistemology, Logic, Methodology, and Philosophy of science, Vol. 306. Dordrecht: Kluwer.

45. Veldman, W. (2009). Brouwer's approximate fixed-point theorem is equivalent to Brouwer's fan theorem. In Lindström, S., Palmgren, E., Segerberg, K., Stoltenberg-Hansen, V. (Eds.) Logicism, intuitionism, and formalism: what has become of them? (pp. 277-299). Synthese Library: Studies in Epistemology, Logic, Methodology, and Philosophy of science, Vol. 341: Springer.

Publisher's Note Springer Nature remains neutral with regard to jurisdictional claims in published maps and institutional affiliations. 